\title{
Global axisymmetric dynamics of thin viscous accretion disks ${ }^{\star}$
}

\author{
O. M. Umurhan ${ }^{1,3}$, A. Nemirovsky ${ }^{1}$, O. Regev ${ }^{1,2,4,5}$, and G. Shaviv ${ }^{1,2}$
}

\author{
1 Department of Physics, Technion-Israel Institute of Technology, 32000 Haifa, Israel \\ e-mail: mumurhan@physics.technion.ac.il \\ 2 Asher Space Research Institute, Technion-Israel Institute of Technology, 32000 Haifa, Israel \\ 3 Department of Astronomy, City College of San Francisco, San Francisco, CA 94112, USA \\ 4 Department of Astrophysics, American Museum of Natural History, New York, NY 10024, USA \\ 5 Department of Astronomy, Columbia University, New York, NY 10028, USA
}

Received 1 August 2005 / Accepted 22 September 2005

\section{ABSTRACT}

The purpose of this paper is to explore the steady state and dynamical behavior of thin, axisymmetric, viscous accretion disks. To facilitate an analytical treatment we replace the energy equation with a general polytropic assumption. The asymptotic expansion of Kluźniak \& Kita (2000, Three-dimensional structure of an alpha accretion disk [arXiv: astro-ph/0006266]), which extended the method of Regev (1983, A\&A, 126, 146) to a full steady polytropic disk (with $n=3 / 2$ ), is further developed and implemented for both the steady (for any polytropic index) and time-dependent problems. The spatial form and temporal behavior of selected dynamical disturbances are studied in detail. It is shown that the transient dynamics resulting from initial perturbations on the linearly stable steady state gives rise to substantial growth of perturbations. We identify the initial perturbation space which leads to such transient growth and provide analytical solutions which manifest this behavior three terms (physical causes) responsible for the appearance of transient dynamics are identified. Two depend explicitly on the viscosity while the third one is relevant also for inviscid disks. The main conclusion we draw is that transient dynamics and, in particular, significant perturbation energy amplification occurs in disks on a global scale. We speculate on the possible implications of these findings to accretion disk theory.

Key words. hydrodynamics - accretion, accretion disks

\section{Introduction}

It is widely accepted that accretion disks form whenever a sufficiently cool gas, endowed with a significant amount of angular momentum, is gravitationally attracted by a relatively compact object. This situation is quite common in astrophysics and, thus, the observational and theoretical study of accretion disks has been intensive (see e.g. Pringle 1981; Frank et al. 2002, for an overview).

At the outset when accretion disks were theoretically proposed (Shakura \& Sunyaev 1973; Lynden-Bell \& Pringle 1974) it had been recognized that the angular momentum transport necessary for accretion cannot take place unless some sort of enhanced transport process operates (typical values of the microscopic viscosity coefficient are far too small to explain the observationally inferred accretion rates). As is well known, fluid turbulence induces anomalous transport and therefore angular momentum transport with the help of turbulent eddy viscosity has been proposed to operate in accretion disks. To date, a detailed theoretical understanding of turbulence and the

* Research supported by the Israel Science Foundation, the Helen and Robert Asher Fund and the Technion Fund for the Promotion of Research. transition to it is still lacking. Consequently, the effective viscosity in disks has usually been approached in a phenomenological way: by parameterizing the effective viscous coefficient $(\alpha)$ on the basis of dimensional arguments. This simple approach has been exceptionally fruitful, giving rise to successful interpretations of most observational results (see Lin \& Papaloizou 1996; Frank et al. 2002, for reviews). In some cases (e.g. dwarf nova models), however, more complex (and cumbersome) prescriptions for the viscosity have been employed.

Until the early 1990's the question what is the physical origin of turbulence (or, more precisely, the anomalous angular momentum transport) in accretion disks has essentially remained open. Robust hydrodynamical stability criteria (e.g. those named after Solberg-Hoiland and Rayleigh), when applied to thin Keplerian accretion disks, indicate that these structures are linearly stable. Moreover, no numerical hydrodynamical calculation had given any indication that Keplerian shear flows are either unstable or can maintain longtime dynamical activity.

The magneto-rotational instability, which has been shown by Balbus \& Hawley (1991) to operate in accretion disk flows (when the fluid is electrically conducting and for not too large initial magnetic fields), provides a natural linear instability 
mechanism for accretion disks and has since become accepted as the driver for magnetohydrodynamic turbulence and the resulting angular momentum transport. It has thus become the operating paradigm in the astrophysical community that purely hydrodynamical turbulence in Keplerian disks is altogether ruled out - this conclusion is based largely on the paucity of purely hydrodynamic turbulent activity in numerical simulations (see Balbus 2003, for a review and references).

Nonetheless, efforts to find a purely hydrodynamical transition to turbulent activity in thin accretion disks have continued until the present time, close to a decade after Balbus et al. (1996) appeared to settle the matter. These have largely been motivated by the fact that a purely hydrodynamical accretion disk flow has a Reynolds number (if based on microscopic viscosity) of the order of $10^{14}$ or so, a quite amazing setting for a hydrodynamically stable flow. Though we shall not deal directly with this issue here, we briefly mention two such recent avenues of exploration.

In the first one, possible linear instabilities of shearing flows in which gravitational stratification plays a catalyzing rôle have been suggested. These stratorotational instabilities (Dubrulle 2004), which are distinct from instabilities giving rise to buoyant convection, have been identified from linear stability analysis (Molemaker et al. 2001; Yavneh et al. 2001; Dubrulle et al. 2004; Shalybkov \& Rudiger 2005; Umurhan 2005) and have been proposed to operate in Keplerian disks. Yavneh et al. (2001) numerically demonstrated that such effects leads to significant non-linear activity in small-gap limit simulations of Taylor-Couette flows in a uniform gravitational field.

The second idea is based on the application to disk flows of the viewpoint, familiar to the fluid-dynamical community, that transient dynamics induced by perturbations of otherwise linearly stable flows may play an important rôle in nonlinearly shaping the final dynamical state. Transient dynamics (specifically, growth of disturbances) has been discussed in the astrophysical literature within two quite different (but related) contexts:

- disturbances experiencing large enough transient growth can trigger a subcritical nonlinear transition into turbulence in a linearly stable shear flow, i.e. via the so-called bypass transition (see e.g. the recent book by Criminale et al. 2003);

- global transient disturbances can be excited either by some external agent or by the pre-existing 3D turbulence itself, however weak, and give rise to intense complex dynamical activity (e.g. Ioannaou \& Kakouris 2001; and see below).

The present paper aims at contributing mainly to the latter aspect, but for the sake of clarity we shall now briefly discuss the former one.

Linear stability analysis of many basic flows (e.g. shear flows) reveal that the relevant operator governing its evolution is non-normal, and hence not self-adjoint. A typical modal analysis resulting from the solution of a boundary value problem given by such an operator may provide only an incomplete description for the original initial value problem. This is because such non-normal problems predict an eigenvalue spectrum which might be continuous, or the eigenfunctions are non-orthogonal, or even imply solutions which are unobtainable analytically. It means that a non-modal approach, i.e. solving the initial value problem, is necessary to analyze the transient dynamics. For example, if sufficient transient growth of an initial disturbance exists in an otherwise linearly stable shear flow, then it may give rise to the kind of nonlinear interactions which can sustain dynamical activity. To save space, we refer the reader to expositions of these ideas in, the book by Criminale et al. (2003), in the book by Schmid \& Henningson (2001), the review article of Grossmann (2000, which focuses on the well known paradigms of shear flows) and to many of the references therein these works. We stress, however, that the rôle of transient dynamics in triggering self-sustained turbulence is not yet well understood.

Among the papers appearing in the astrophysical literature Ioannaou \& Kakouris (2001) were the first to apply the concept of transient dynamics (and specifically growth) to accretion disk flows. Most subsequent astrophysical studies explored transient growth within the context of the local shearing box approximation $^{1}$ (Goldreich \& Lynden-Bell 1965; Balbus et al. 1996). The consensus seems to be that the transient dynamic phase of linearized disturbances can exhibit substantial growth of initial perturbations for an array of cases (Chagelishvili et al. 2003; Tevzadze et al. 2003; Yecko 2004; Mukhopadhyayi et al. 2005; Ashfordi et al. 2005).

Interest in the scope and severity of a transiently growing initial disturbance lies in the more basic question as to whether or not there exists a minimum amplitude that must be achieved by such a disturbance before it can either trigger or somehow drive sustained dynamical activity. If such minimum criteria exist then it might also imply that there are minimum Reynolds numbers for such transitions to take place. As of yet this matter remains largely open. However, Ashfordi et al. (2005) estimate that the flow be at least $\operatorname{Re}>10^{5}$ in order for transiently growing linear modes to achieve sufficient strength so as to enable nonlinear sustained activity, e.g. a turbulent dynamical state. It would seem reasonable to suppose, then, that very high resolution 3D numerical simulations are called for (Longaretti 2001). In two dimensions, the simulations of Umurhan \& Regev (2004), done within the shearing box approximation, indicate that dynamical activity is sustained for very long times by what seems to be recurrent transient growth episodes, for Reynolds numbers determined by the numerical "viscosity" of their spectral code $\left(\sim 10^{5}\right)$. Very recently, Lesur \& Longaretti (2005) have explicitly demonstrated, using high resolution 3D numerical simulations, that a subcritical transition to turbulence does exist at very high Reynolds numbers. The Coriolis force does not appear to quench such a transition as it was claimed before, e.g. Balbus et al. (1996) and Balbus et al. (1999). However, it appears the efficiency of turbulent transport in such subcritical flows is insufficient for astrophysical purposes (i.e. accretion disks). Thus, it seems that the excitation of global secondary flows atop the weak turbulent state may be required for the necessary transport.

\footnotetext{
${ }^{1}$ See Appendix A of Umurhan \& Regev (2004) for a systematic discussion of this approximation.
} 
Irrespective of the controversial nature regarding the source of disk turbulence and its corresponding effective viscosity, the structure and dynamics of viscous disks, even with the simplest $\alpha$ viscosity prescription, still remain of interest. Numerous applications of the $\alpha$-disk paradigm, from young stellar objects, through close binary stars, and up to active galactic nuclei, abound in the literature. It is impractical to cite here even the most recent theoretical studies, both numerical and analytical, on the structure and dynamics of accretion disks in various systems. We refer the reader to the review paper of Papaloizou \& Lin (1995) and the recent graduate text by Vishniac \& Park (2005) and references therein.

In this paper we shall investigate the dynamical (i.e. time dependent) behavior of thin, viscous accretion disks, assuming axisymmetry, but allowing for vertical structure. We shall choose the simplest viscosity prescription (the $\alpha$ model) and examine the dependence of our results on the value of this parameter. Exploiting the fact that the disk is very thin, we shall attempt to obtain analytically approximate time-dependent solution to the relevant fluid-dynamical equations. Such an asymptotic approach was introduced for the first time to the study of thin viscous accretion disks by Regev (1983) in the context of accretion disk boundary layers. It was later further developed and used in a remarkable analytical work by Kluźniak \& Kita (2000) to solve for the steady structure of a polytropic viscous axisymmetric 3D disk. This study revealed the presence of a steady meridional flow pattern with backflow for values of the $\alpha$ less than some critical value. This result and feature was confirmed by Regev \& Gitelman (2002), who abandoned the polytropic assumption and included an energy equation (employing the diffusion approximation in the treatment of the vertical radiative transport).

The purpose of the present paper is to extend the global asymptotic approach of Kluźniak \& Kita (2000) to the timedependent case. This method will then be employed in the pursuit of axisymmetric dynamics of thin viscous disks and the identification of the terms (mechanisms) responsible for the assortment of resulting behavior, including any transient dynamics. Recently, Umurhan \& Shaviv (2005) employed such a global asymptotic approach in the study of the dynamical evolution of global axisymmetric initial disturbances in an inviscid 3D disk. They obtained algebraic growth in the transient dynamics, a behavior that can be only sustained for some initial period, because if the disturbances become to large the asymptotic expansion breaks down. It is important to note that the asymptotic approach to the dynamics of accretion disks is not equivalent to a linear stability analysis, even though the initial disturbance (over a basic steady state) used may be small. Whereas the one is strictly linear and infinitesimal the other is finite amplitude and, in principle, nonlinear (see Umurhan \& Shaviv 2005, and below in the last section). As we shall see, the secondary meridional flows found, for small enough $\alpha$, in steady state (Kluźniak \& Kita 2000; Regev \& Gitelman 2002) persist also in the time dependent case along with transient spatio-temporal complexity.

As we said, the primary tool utilized here in order to facilitate an analytical treatment is the asymptotic expansion, where the dependent variables and governing equations are expanded in powers of some small quantity (here the measure of the disk's "thinness", $\epsilon$ - see below). Exposing the resulting mathematical system (an initial value problem) to a set of initial conditions, in which the extreme geometry of the disk structure is taken into account (i.e. its "thinness"), we are aiming to obtain an analytically treatable problem. To achieve this goal we also assume a polytropic relation between the pressure and density. Since this assumption was found to make little substantive difference to the steady meridional flow solution (Regev \& Gitelman 2002; and cf. Kluźniak \& Kita 2000), we see its use as justified here as well. We emphasize again that we shall consider viscous flow using the standard $\alpha$ prescription. Thus we actually assume that some kind of effective viscosity producing mechanism is already operative. The effective Reynolds number of the flow investigated here is thus $\operatorname{Re}_{\text {eff }} \sim 1 / \alpha$.

The advantage of the asymptotic method and approach and the simplistic polytropic assumption is obvious - the treatment will be analytical and the responsible physical effects leading to any interesting dynamics may be transparently traced. It is clear, however, that the present analytic analysis should be ultimately complemented with a detailed and uncompromising numerical solution and a proper treatment of energy generation and transfer.

This paper is organized in the following way. In Sect. 2 we state all our assumptions, derive the basic non-dimensional equations and introduce the asymptotic expansions for the dependent variables that are used in this work. Although we are bound to repeat here some previously published (and unpublished) work, we feel that explaining the global asymptotic approach to thin accretion disks (here including also time dependence) in a self-contained way may be useful for better understanding of our work here as well as perhaps for future investigations. In Sect. 3 the steady solutions of the equations are given and discussed. These solutions are later used to study the dynamical evolution by adding (in a suitable order) a time dependent disturbance to them. The dynamics of the disturbances, within the context of an initial value problem, is the subject of Sect. 4, where we present the results and discuss their nature. A global principle as well as detailed dynamical behavior and some limits are discussed in the final section, in an effort to uncover the origin of our primary result - the appearance of nontrivial transient dynamics. We conclude with a discussion on the possible relevance of our results to the behavior of real accretion disks. The case of a general polytropic index and some technical details of the analytical asymptotic procedure needed for obtaining the solutions are given in the Appendices.

\section{Assumptions, non dimensional equations and asymptotic expansions}

Our starting point are the general Navier-Stokes equations

$$
\begin{aligned}
& \rho \frac{\partial \boldsymbol{V}}{\partial t}+\rho(\boldsymbol{V} \cdot \nabla) \boldsymbol{V}=-\nabla P+\rho \boldsymbol{b}+\nabla \cdot \boldsymbol{\sigma}, \\
& \frac{\partial \rho}{\partial t}+\nabla \cdot(\rho \boldsymbol{V})=0
\end{aligned}
$$

where $\boldsymbol{V}$ is the three dimensional velocity vector, $\rho$ is the density, $P$ is the pressure and $\boldsymbol{b}$ is the body force per unit mass. 
The Cartesian components of the viscous stress tensor, $\sigma$ are given by

$\sigma_{j k}=\eta\left(\frac{\partial V_{j}}{\partial x_{k}}+\frac{\partial V_{k}}{\partial x_{j}}-\frac{2}{3} \delta_{j k} \nabla \cdot V\right)$,

in which $\eta$ is the dynamic viscosity coefficient (we neglect bulk viscosity because the processes discussed here are sufficiently slow to take place under thermodynamic equilibrium).

In the context of accretion disks it is natural to present the equations in cylindrical polar coordinates with $r, z$ and $\phi$ being the radial, vertical and azimuthal coordinates respectively. Also, the disk matter's self gravity is neglected and therefore the body force derives from the gravitational potential of a central accreting object, whose mass is $M$, say. Thus we have $\boldsymbol{b}=-\nabla \Phi$ with

$\Phi=-\frac{G M}{\sqrt{r^{2}+z^{2}}}$.

Regarding the viscosity of the accretion disk flow, we shall assume that the situation is such that the viscosity coefficient is greatly enhanced relatively to the microscopic one (see above in the Introduction). We will thus use one of the standard $\alpha$-prescriptions (see below). If this effective viscosity enhancement results from $\eta$ actually being the eddy viscosity of an already turbulent flow, the variables of the fluid dynamical equations should be understood as mean quantities obtained by Reynolds averaging, a standard technique in treatments of turbulence (e.g. Monin \& Yaglom 1971).

We proceed in a manner similar to that of Kluźniak \& Kita (2000), hereafter KK, and Regev \& Gitelman (2002), hereafter RG, by writing the equations in cylindrical coordinates (with the assumption of axisymmetry) in their non-dimensional form, which allows analytical asymptotic treatment. The difference is that here we shall not assume a steady flow: we retain the time derivative terms. This will enable us to analyze the dynamical evolution of deviations from the steady KK solution.

\subsection{The scalings and additional assumptions}

We consider here cold disks. Physically this means that the characteristic disk height (as measured from the midplane) is much smaller than its characteristic radius as measured from the accreting star's center. This feature is readily brought to the fore when we scale the dependent variables of the problem by their characteristic values (see e.g. Regev 1983, hereafter R), which we shall denote by the "tilde" sign. With $\tilde{R}$ the characteristic radial scale of the disk (which is also a natural unit of the radial coordinate), the natural scaling for the angular velocity is the Keplerian value $\tilde{\Omega} \equiv \Omega_{k}(\tilde{R})=\left(G M / \tilde{R}^{3}\right)^{1 / 2}$. Also, the density will be scaled by a characteristic value (say that at the radius $\tilde{R}$ and at the midplane of the disk): $\tilde{\rho}$.

We assume throughout this work that the disk equation of state is polytropic - both in steady and dynamical states. This means that we can assume that the pressure and density always obey the relationship $P=P(\rho) \equiv K \rho^{(1+1 / n)}$, where $n$ is the (constant) polytropic index and $K$ is a constant. It follows that the typical scale of the pressure is $\tilde{P}=P(\tilde{\rho})$. Also, we choose the typical sound speed to be $\tilde{c}_{\mathrm{s}}=\sqrt{\tilde{P} / \tilde{\rho}}$. The assumption that the disk is cold means that $\tilde{c}_{\mathrm{s}} \ll \tilde{R} \tilde{\Omega}$ which, in turn, allows for the definition of the vertical scale height of the disk, $\tilde{H} \equiv \tilde{c}_{\mathrm{s}} \tilde{\Omega}$. This is thus the natural ${ }^{2}$ scale for the coordinate $z$. Formally speaking, we may define the small expansion parameter $\epsilon$ to be,

$\epsilon \equiv \frac{\tilde{c}_{\mathrm{s}}}{\tilde{R} \tilde{\Omega}}=\frac{\tilde{H}}{\tilde{R}} \ll 1$.

This disparity of scales is exploited in what follows. The radial $\left(V_{r}\right)$ and vertical $\left(V_{z}\right)$ velocities are scaled by the typical sound speed $\tilde{c}_{\mathrm{s}}$ and the angular velocity $\left(\Omega=V_{\phi} / r\right)$ by $\Omega$. Furthermore, we find that there is one natural choice for the temporal scaling, since a typical rotation time $\tilde{\Omega}^{-1}$ and the vertical sound crossing time, $\tilde{H} / \tilde{c}_{\mathrm{s}}$, are defined to be identical.

From here on all variables are assumed to be nondimensionalized according to what has just been described. Thus the Keplerian angular velocity and the polytropic relations for the pressure and sound speeds are

$$
\begin{aligned}
& \Omega_{k}=\frac{1}{r^{3 / 2}}, \quad P=\rho^{1+1 / n}, \\
& c_{\mathrm{s}}^{2}=\frac{\mathrm{d} P}{\mathrm{~d} \rho}=\left(1+\frac{1}{n}\right) \frac{P}{\rho}=\left(1+\frac{1}{n}\right) \rho^{1 / n} .
\end{aligned}
$$

To be consistent with previous works (e.g. KK) we make use of the non-dimensional sound speed $c_{\mathrm{s}}$ as the dependent variable instead of the pressure $P$. Consequently, we replace the pressure gradient terms in the equations in the following way:

$\frac{1}{\rho} \frac{\partial P}{\partial r}=n \frac{\partial c_{\mathrm{s}}^{2}}{\partial r}, \quad \frac{1}{\rho} \frac{\partial P}{\partial z}=n \frac{\partial c_{\mathrm{s}}^{2}}{\partial z}$.

The standard $\alpha$ model of Shakura \& Sunyaev (1973) is based on the assumption that the only non vanishing viscous stress component is $\sigma_{r \phi}$ and it is proportional to the pressure. Following KK, we adopt this assumption and derive from it the form of viscosity coefficient, but include in the dynamical equations all the components of the stress tensor. In lowest order in $\epsilon$ the angular velocity of a disk is Keplerian and we get (with the dynamic viscosity coefficient scaled by $\tilde{c}_{\mathrm{s}} \tilde{H}$ ) the nondimensional relation (see also RG)

$\eta=\frac{2}{3} \frac{\alpha P}{\Omega_{k}}=\frac{2}{3} \frac{\alpha c_{\mathrm{s}}^{2} \rho}{\Omega_{k}\left(1+\frac{1}{n}\right)}$,

where $\alpha$ is the viscosity parameter.

\subsection{Nondimensional equations and asymptotic expansions}

As stated in the Introduction, we seek axisymmetric solutions (in both the steady and dynamical cases) of the equations of motion. Denoting the non-dimensionalized radial and vertical velocities as $u$ and $v$, respectively, the equations now appear as they do in KK and RG except that here we allow for

\footnotetext{
2 Note that though a "cold" disk implies $H / R \ll 1$, a "hot" disk, i.e. one where $\epsilon \gg 1$, does not imply that $H / R \gg 1$. Instead it just means that the structure starts looking more like a star with $H / R \sim 1$.
} 
time-dependence (the time unit is $1 / \tilde{\Omega}$ ). Consequently timederivatives are included and all the dependant variables are functions of $r, z$ and $t-$

$$
\begin{aligned}
\epsilon \frac{\partial u}{\partial t}+\epsilon^{2} u \frac{\partial u}{\partial r}+\epsilon v \frac{\partial u}{\partial z}-\Omega^{2} r= \\
\quad-\frac{1}{r^{2}}\left[1+\epsilon^{2} \frac{z^{2}}{r^{2}}\right]^{-3 / 2}+\frac{\epsilon}{\rho} \frac{\partial}{\partial z}\left(\eta \frac{\partial u}{\partial z}\right) \\
+\epsilon^{2}\left[-n \frac{\partial c_{\mathrm{s}}^{2}}{\partial r}+\frac{1}{\rho} \frac{\partial}{\partial z}\left(\eta \frac{\partial v}{\partial r}\right)-\frac{1}{\rho} \frac{\partial}{\partial r}\left(\eta \frac{2}{3} \frac{\partial v}{\partial z}\right)\right] \\
+\epsilon^{3}\left[-\frac{2 \eta u}{\rho r^{2}}+\frac{1}{\rho r} \frac{\partial}{\partial r}\left(2 \eta r \frac{\partial u}{\partial r}\right)-\frac{1}{\rho} \frac{\partial}{\partial r}\left(\frac{2}{3} \frac{\eta}{r} \frac{\partial(r u)}{\partial r}\right)\right],
\end{aligned}
$$

$$
\begin{aligned}
\rho \frac{\partial \Omega}{\partial t}+ & \frac{\rho u}{r^{2}} \frac{\partial\left(r^{2} \Omega\right)}{\partial r}+\rho v \frac{\partial \Omega}{\partial z}= \\
& \frac{\partial}{\partial z}\left(\eta \frac{\partial \Omega}{\partial z}\right)+\epsilon^{2} \frac{1}{r^{3}} \frac{\partial}{\partial r}\left(\eta r^{3} \frac{\partial \Omega}{\partial r}\right)
\end{aligned}
$$

$$
\begin{aligned}
& \frac{\partial v}{\partial t}+\epsilon u \frac{\partial v}{\partial r}+v \frac{\partial v}{\partial z}= \\
& -\frac{z}{r^{3}}\left(1+\epsilon^{2} \frac{z^{2}}{r^{2}}\right)^{-3 / 2}-n \frac{\partial c_{\mathrm{s}}^{2}}{\partial z}+\frac{4}{3} \frac{1}{\rho} \frac{\partial}{\partial z}\left(\eta \frac{\partial v}{\partial z}\right) \\
& +\epsilon \frac{1}{\rho}\left[\frac{1}{r} \frac{\partial}{\partial r}\left(\eta r \frac{\partial u}{\partial z}\right)-\frac{2}{3} \frac{\partial}{\partial z}\left(\frac{\eta}{r} \frac{\partial(r u)}{\partial r}\right)\right]+\epsilon^{2} \frac{1}{\rho r} \frac{\partial}{\partial r}\left(\eta r \frac{\partial v}{\partial r}\right), \\
& \frac{\partial \rho}{\partial t}+\frac{\epsilon}{r} \frac{\partial(r \rho u)}{\partial r}+\frac{\partial(\rho v)}{\partial z}=0,
\end{aligned}
$$

where the gravitational potential of the central star has been expanded only up to second order in $\epsilon$.

We seek asymptotic solutions to Eqs. (9)-(12) by writing all the dependent variables in a power series of the small parameter $\epsilon$, i.e. we formally write

$\boldsymbol{U}(r, z, t)=\sum_{m=0}^{\infty} \epsilon^{m} \boldsymbol{U}_{m}$,

where $\boldsymbol{U}=\left(u, v, \Omega, c_{\mathrm{s}}^{2}\right)^{\mathbf{T}}$. We remind the reader that $c_{\mathrm{s}}$ and $\rho$ are not independent (on account of the assumption of polytropes) and are related through Eq. (6).

It has been shown before (see R, KK, RG) that in steady state the lowest order nonzero components of the meridional velocities are $u_{1}$ and $v_{2}$ and, in addition, the choice $\Omega_{1}=\rho_{1}=0$ (and thus also $c_{\mathrm{s} 1}=0$ ) can be consistently made. Guided by these results (see also below) we retain in the expansions for $\Omega$, $c_{\mathrm{s}}^{2}, \rho$ and $v$ only even powers of $\epsilon$ (in the case of $v$ starting from $v_{2}$ ), while for the $u$ expansions only odd powers are kept, starting with $u_{1}$,

$$
\begin{aligned}
\Omega(r, z, t)= & \Omega_{0}(r, z)+\epsilon^{2}\left[\Omega_{2}(r, z)\right. \\
& \left.+\Omega_{2}^{\prime}(r, z, t)\right]+\epsilon^{4}\left[\Omega_{4}+\Omega_{4}^{\prime}(r, z, t)\right]+\cdots \\
u(r, z, t)= & \epsilon\left[u_{1}(r, z)+u_{1}^{\prime}(r, z, t)\right] \\
& +\epsilon^{3}\left[u_{3}(r, z)+u_{3}^{\prime}(r, z, t)\right]+\cdots \\
v(r, z, t)= & \epsilon^{2}\left[v_{2}(r, z)+v_{2}^{\prime}(r, z, t)\right] \\
& +\epsilon^{4}\left[v_{4}(r, z)+v_{4}^{\prime}(r, z, t)\right]+\cdots
\end{aligned}
$$

$$
\begin{aligned}
c_{\mathrm{S}}^{2}(r, z, t)= & c_{\mathrm{s} 0}^{2}(r, z)+\epsilon^{2}\left[c_{\mathrm{s} 2}^{2}(r, z)+c_{\mathrm{s} 2}^{2}(r, z, t)\right] \\
& +\epsilon^{4}\left[c_{\mathrm{s} 4}^{2}(r, z)+c_{\mathrm{s} 4}^{\prime}(r, z, t)\right]+\cdots \\
\rho(r, z, t)= & \rho_{0}(r, z)+\epsilon^{2}\left[\rho_{2}(r, z)+\rho_{2}^{\prime}(r, z, t)\right] \\
& +\epsilon^{4}\left[\rho_{4}(r, z)+\rho_{4}^{\prime}(r, z, t)\right]+\cdots
\end{aligned}
$$

All $O$ (1) quantities are assumed to be time-independent (in accord with the steady state results of RG and KK). As is also apparent, we have assumed no meridional flow at $O(1)$ since, as said above, it has been shown before that $u_{0}=v_{0}=v_{1}=0$. Time dependence has been introduced into the expansions at $O\left(\epsilon^{2}\right)$ for the functions $\Omega, \rho, c_{\mathrm{s}}^{2}$ and $v$ while it appears at $O(\epsilon)$ for $u$. At all orders in which time dependence is introduced we split the solutions up into a sum of a steady solution and a dynamical one, denoted by a prime, and this is true for all high orders as well. Thus, from now and on all the terms of the dependent variables expansions that are dependent on time are denoted by a prime, while those without prime are just space dependent, that is, steady. The implicit assumption in this kind of splitting is that the time-dependent part is a perturbation (not necessarily infinitesimal!) on the steady state. In any case, the expansions Ansatz (14)-(18) can always be considered as a particular choice of a perturbation kind.

The steady part of the solutions up to second order will be identical to the solutions obtained in KK and RG (for $n=3 / 2$ ). In this work we shall limit the analysis of the evolution of the time-dependent disturbances to terms of order $\epsilon^{2}$ or lower.

\section{Steady state}

\section{1. $O(1)$}

Neither the techniques nor the qualitative implications of this section differ from the results of KK. All that is substantively different is that the steady solutions are here derived in terms of an arbitrary polytropic index $n$.

The lowest order equations are straightforwardly solved to yield solutions quite well-known in the literature (see, e.g., Hoshi 1977). These are,

$$
\begin{array}{ll}
c_{\mathrm{s} 0}^{2}(r, z)=\frac{h^{2}(r)-z^{2}}{2 n r^{3}}, & \Omega_{0}=\Omega_{k}=\frac{1}{r^{3 / 2}}, \\
\rho_{0}(r, z)=\left[\frac{c_{\mathrm{s} 0}^{2}(r, z)}{1+\frac{1}{n}}\right]^{n} . &
\end{array}
$$

At $z=h(r), c_{\mathrm{s} 0}^{2}=\rho_{0}=0$, thus $h(r)$ is explicitly determined when solving the next order steady state equations. For values of $|z| \geq h, c_{\mathrm{s} 0}^{2}, \rho_{0}$ and all other associated quantities remain zero. Additionally the relationships between the sound speed, pressure and density, as well as their vertical gradients, are given by,

$$
\frac{P_{0}}{\rho_{0}}=\frac{c_{\mathrm{s} 0}^{2}}{1+\frac{1}{n}}, \quad \frac{1}{\rho_{0}} \frac{\partial P_{0}}{\partial z}=n \frac{\partial c_{\mathrm{s} 0}^{2}}{\partial z}=-\frac{z}{r^{3}} .
$$

It useful to note that we shall use in the equations to all orders only the zeroth order value of the viscosity coefficient, that is, will not actually consider it as an expandable function, 
but rather as a prescribed function which is based on the zeroth order of the pressure. Thus

$\eta \equiv \eta_{0}=\frac{2}{3} \alpha P_{0} r^{3 / 2}$

\section{2. $O\left(\epsilon^{2}\right)$}

The steady-state equations at the next non-trivial order of $\epsilon$ are,

$$
\begin{aligned}
& n \frac{\partial c_{\mathrm{s} 0}^{2}}{\partial r}-\frac{3}{2} \frac{z^{2}}{r^{4}}=2 \Omega_{0} \Omega_{2} r+\frac{1}{\rho_{0}} \frac{\partial}{\partial z}\left(\eta \frac{\partial u_{1}}{\partial z}\right), \\
& -\frac{1}{r^{3} \rho_{0}} \frac{\partial}{\partial r}\left(\eta r^{3} \frac{\partial \Omega_{0}}{\partial r}\right)=-\frac{u_{1}}{r^{2}} \frac{\partial\left(r^{2} \Omega_{0}\right)}{\partial r}+\frac{1}{\rho_{0}} \frac{\partial}{\partial z}\left(\eta \frac{\partial \Omega_{2}}{\partial z}\right), \\
& 0=\frac{1}{r} \frac{\partial}{\partial r}\left(r \rho_{0} u_{1}\right)+\frac{\partial}{\partial z}\left(\rho_{0} v_{2}\right), \\
& 0=\frac{3}{2} \frac{z^{3}}{r^{5}}-n \frac{\partial c_{\mathrm{s} 2}^{2}}{\partial z}+\frac{4}{3} \frac{1}{\rho_{0}} \frac{\partial}{\partial z}\left(\eta \frac{\partial v_{2}}{\partial z}\right) \\
& \quad+\frac{1}{\rho_{0}}\left[\frac{1}{r} \frac{\partial}{\partial r}\left(\eta r \frac{\partial u_{1}}{\partial z}\right)-\frac{2}{3} \frac{\partial}{\partial z}\left(\frac{\eta}{r} \frac{\partial\left(r u_{1}\right)}{\partial r}\right)\right] .
\end{aligned}
$$

These equations have solutions exhibiting a steady meridional flow, something which we will formalize below.

KK analytically found such solutions at this order (i.e. Eqs. (22)-(25)) for the polytropic index $n=3 / 2$. We discuss in considerable detail the solutions for an arbitrary polytropic index $n$ in Appendix A. Here, we would only like to stress some important points.

First, as KK demonstrated, there is a value of $\alpha$ below which there is a certain amount of backflow in the disk, that is, a stagnation radius exists, beyond which the radial velocity, near the disk midplane, is directed outwards.

Second, also as KK demonstrated, the disk height as a function of $r, h(r)$, depends on the mass accretion rate, which in the appropriate units turns out to be of order $\epsilon, \dot{M}=\epsilon \dot{M}_{1}$, and the polytropic index $n$. The disk's height $h(r)$ can be derived in an asymptotically valid way only for radii significantly larger than $r_{*}$, where $r_{*}$ is the zero torque position, i.e. the point at which the angular velocity (having the Keplerian value at lowest order) must pass a maximum on the way to matching it to some inner boundary value (the angular velocity of the surface of the accreting object, which is sub-Keplerian). The point, if treated carelessly, will produce pathologies like zero disk thickness, a zero density and divergent velocities. To find the structure near $r_{*}$, some boundary layer treatment, using singular perturbation methods like the ones employed by, for example, Bertout \& Regev (1995, see also references therein) or fully numerical approaches (see Popham \& Narayan 1995, and references therein), must be employed. Thus in this work, as well as in KK and RG, we are dealing with the disk only for radii sufficiently larger than $r_{*}$.

Third, these solutions do not allow for a detailed outer boundary condition (only an integral condition for the constancy of the total mass influx in the disk is employed). Thus, the KK solution and our generalization thereof (both steady and dynamical) are valid in regions of the disk that are not too close to its edges in the radial direction.
Finally, it is an interesting result that in the expression for the disk height for arbitrary polytropic index (see Eq. (A.5)) the disk flares for $n>3 / 2$, namely $h(r) \sim r^{m}$ with $m>1$.

The form of $h(r)$ given in $\mathrm{KK}$ is recovered by substituting $n=3 / 2$ into the general expressions (see Eq. (A.5)) given in Appendix A and reads as follows,

$h(r)=(2 \Lambda)^{\frac{1}{6}} r^{\frac{11}{12}}(\sqrt{r}-\sqrt{r})^{\frac{1}{6}}$,

$\Lambda \equiv \frac{\dot{M}_{1}}{\alpha} \frac{\Gamma(4)}{\Gamma(5 / 2)} \cdot \frac{(5)^{\frac{3}{2}}}{3 \pi^{3 / 2}(11 / 2)}$.

The height of the disk at the fiducial radius $r=1$ is denoted by $h_{1}$ and is

$h_{1} \equiv(2 \Lambda)^{\frac{1}{6}}\left(1-\sqrt{r}_{*}\right)^{\frac{1}{6}}$.

\section{Dynamical evolution}

The expansion procedure outlined in the previous section for the steady state solutions is extended here to dynamical problems. In other words, we allow for time dependence and include it in all equations at successive orders of $\epsilon$ and subject them to appropriate initial conditions. Regarding the radial boundary conditions, we avoid the problems posed by them by considering only the portion of the disk with an inner radius $r_{\text {in }}$ and an outer radius $r_{\max }$, as in the steady-state case (see KK).

In the following, we discuss the vertical boundary conditions which we adopt. Let us say from the outset that the choices we make seem to us to be physically reasonable and, yet, allow for some analytical tractability. In steady-state, the disk lies between $z= \pm h(r)$, in which $h(r)$ is as given in the previous sections. In this work we shall consider the "surface" of the disk, in the time-dependent case, to consist of the last fluid parcel which, in a steady disk, is at $z=h(r)$. Technically speaking, the position of the evolving disk surface should include a time-dependent correction term accounts (to $h(r)$ ). However, to the order up to which equations are sought in this work, it suffices to enforce the boundary conditions at $z=h(r){ }^{3}$

In addition, the equatorial dynamical behavior allows two kinds of dynamical considerations according to whether the disturbances in the disk are sinuous or varicose. Varicose modes have even symmetry about the $z=0$ axis in all the quantities except the vertical velocity which has odd symmetry there. Sinuous modes have opposite symmetry of varicose modes.

Firstly, thus, in this work we consider only varicose modes (the steady state meridional flow has this symmetry as well). However, it should be noted that when mode couplings begin to play a role in the evolution of the system (which occur at orders of $\epsilon$ higher than that explored in this work), sinuous and

\footnotetext{
${ }^{3}$ In other words, the correction term to the disk surface does not alter the resulting disk dynamics at the lowest order for which they are determined. This is similar to the standard procedure applied in the treatment of fluid surfaces (either free or between two fluids) when it is subject to small deviations from a steady state (see e.g. Landau \& Lifshitz 1989, in the case of surface waves or the Kelvin-Helmholtz instability)
} 
varicose modes dynamically influence each other via the nonlinear advection terms in the equation for the vertical velocity, that is, the $v \partial_{z} v$ term in Eq. (11) provides this cross-mode interaction.

Secondly, we allow the disk surface to flutter about freely in a Lagrangian sense. This, in turn, means that we require that the pressure on the moving surface vanish for all times. This, then, leads to the condition that the Lagrangian pressure fluctuation vanishes on this surface i.e.,

$\left(\frac{\partial}{\partial t}+\epsilon u \frac{\partial}{\partial r}+v \frac{\partial}{\partial z}\right) P=0, \quad$ at $z= \pm h(r)$.

Given the polytropic relation (6), the above boundary condition may be simplified with the aid of the mass continuity Eq. (12), to

$P\left(\epsilon \frac{\partial u}{\partial r}+\frac{\partial v}{\partial z}\right)=0$ at $z= \pm h(r)$.

The above is a restatement of the condition that there should be no work performed at the moving boundary.

Thirdly, we posit that the viscous stresses on the surface of the disk are zero. This means that the product of the viscosity coefficient and the velocities (or the velocity gradients) tend to zero on the boundary. Since this is a free surface and the viscosity prescription given tends to zero at zero density, we will impose the more restrictive free-surface condition,

$\lim _{z \rightarrow h} \eta r \hat{\boldsymbol{n}} \cdot \nabla \Omega \rightarrow 0$ and $\eta \hat{\boldsymbol{n}} \cdot \nabla u \rightarrow 0$,

where $\hat{\boldsymbol{n}}$ is the normal to the surface $\boldsymbol{S}$ of the disk. In the event where the surface of the disk is approximately aligned with the normal to the vertical direction this condition becomes,

$\lim _{z \rightarrow h} \eta r \frac{\partial \Omega}{\partial z} \rightarrow 0$ and $\lim _{z \rightarrow h} \eta \frac{\partial u}{\partial z} \rightarrow 0$.

In other words, we allow the gradient of the $u$ and $r \Omega$ velocities to diverge as one approaches the disk height $h$ but the rate thereof should be slower than the rate at which $\eta$ tends to zero. In the solutions that are developed for these modes (see below), we find that this condition is always satisfied and there are never diverging gradients of either $u$ or $\Omega$.

Lastly, we impose that the total vertically integrated mass flux through an annular section of the disk be fixed and steady. In turn this means that the vertically integrated mass flux through an annular disk section of dynamically varying quantities ought to be identically zero, i.e.

$2 \pi r \int_{-\infty}^{\infty} \rho u_{i}^{\prime} \mathrm{d} z=0$

in which $u_{i}^{\prime}(r, z, t)$ are the dynamically varying portions of the radial velocity at each order $i$ of the $\epsilon$ expansion.

\section{1. $O\left(\epsilon^{2}\right)$ dynamical equations}

With the solution expansions implemented into the equation set (9)-(12) we find at the lowest nontrivial order describing the dynamical evolution of the disturbances (denoted by the primed quantities) the following,

$$
\begin{aligned}
\partial_{t} u_{1}^{\prime}= & 2 \Omega_{0} \Omega_{2}^{\prime} r+\frac{1}{\rho_{0}} \frac{\partial}{\partial z}\left(\eta \frac{\partial u_{1}^{\prime}}{\partial z}\right), \\
\partial_{t} \Omega_{2}^{\prime}= & -\frac{u_{1}^{\prime}}{r^{2}} \frac{\mathrm{d}\left(r^{2} \Omega_{0}\right)}{\mathrm{d} r}+\frac{1}{\rho_{0}} \frac{\partial}{\partial z}\left(\eta \frac{\partial \Omega_{2}^{\prime}}{\partial z}\right), \\
\partial_{t} v_{2}^{\prime}= & -n \frac{\partial c_{\mathrm{s} 2}^{2^{\prime}}}{\partial z}+\frac{4}{3} \frac{1}{\rho_{0}} \frac{\partial}{\partial z}\left(\eta \frac{\partial v_{2}^{\prime}}{\partial z}\right) \\
& +\frac{1}{r} \frac{1}{\rho_{0}} \frac{\partial}{\partial r}\left(\eta r \frac{\partial u_{1}^{\prime}}{\partial z}\right)-\frac{2}{3} \frac{1}{\rho_{0}} \frac{\partial}{\partial z}\left[\eta\left(\frac{1}{r} \frac{\partial\left(r u_{1}^{\prime}\right)}{\partial r}\right)\right], \\
\partial_{t} \rho_{2}^{\prime}= & -\frac{1}{r} \frac{\partial}{\partial r}\left(r \rho_{0} u_{1}^{\prime}\right)-\frac{\partial}{\partial z}\left(\rho_{0} v_{2}^{\prime}\right) .
\end{aligned}
$$

The polytropic equation state tells us that the relationship between the second order density and sound speed dynamical disturbances is

$$
c_{\mathrm{s} 2}^{2^{\prime}}=c_{\mathrm{s} 0}^{2} \frac{1}{n} \frac{\rho_{2}^{\prime}}{\rho_{0}} .
$$

This algebraic relation is necessary to complete the linear PDE set (33)-(36).

It is important to note that the above four equations support two sorts of modes, namely a pair comprising of purely vertical disturbances and a second pair containing disturbances in all the velocity components. The nature of these modes is detailed below. Such a classification is possible because Eqs. (33), (34) dynamically decouple from Eqs. (35), (36), i.e. the quantities $u_{1}^{\prime}$ and $\Omega_{2}^{\prime}$ are not dynamically influenced by $v_{2}^{\prime}$ and $\rho_{2}^{\prime}$, while the converse is not true. That is, disturbances in $u_{1}^{\prime}$ and $\Omega_{2}^{\prime}$ will drive a dynamical response in $v_{2}^{\prime}$ and $\rho_{2}^{\prime}$. This mathematical structure is reminiscent of the behavior of the steady-state equations (see Sect. 3.2 and KK).

To be more specific Eqs. (33), (34) and (35), (36) may be combined to give two second order equations for $u_{1}^{\prime}$ and $v_{2}^{\prime}$,

$\mathcal{P} u_{1}^{\prime}=0$,

$\mathcal{L} v_{2}^{\prime}=\left[\partial_{t} \mathcal{F}+\mathcal{G}\right] u_{1}^{\prime}$,

in which the operators are,

$\mathcal{P} \equiv\left(\partial_{t}-\frac{1}{\rho_{0}} \frac{\partial}{\partial z} \eta \frac{\partial}{\partial z}\right)^{2}+\Omega_{0}^{2}$,

$\mathcal{L} \equiv \partial_{t}^{2}-c_{\mathrm{s} 0}^{2} \frac{\partial^{2}}{\partial z^{2}}-(n+1) \frac{\partial c_{\mathrm{s} 0}^{2}}{\partial z} \frac{\partial}{\partial z}$

$$
-n \frac{\partial^{2} c_{\mathrm{s} 0}^{2}}{\partial z^{2}}-\frac{4}{3} \frac{1}{\rho_{0}} \frac{\partial}{\partial z} \eta \frac{\partial}{\partial z} \partial_{t},
$$

$\mathcal{F} \equiv-\frac{2}{3} \frac{1}{r \rho_{0}} \frac{\partial}{\partial z} \eta \frac{\partial}{\partial r} r+\frac{1}{r \rho_{0}} \frac{\partial}{\partial r} r \eta \frac{\partial}{\partial z}$,

$\mathcal{G} \equiv \frac{\partial}{\partial z} \frac{c_{\mathrm{s} 0}^{2}}{r \rho_{0}} \frac{\partial}{\partial r} \rho_{0} r$

The zero Lagrangian pressure condition gives, at this order,

$P_{0}\left(\frac{\partial u_{1}^{\prime}}{\partial r}+\frac{\partial v_{2}^{\prime}}{\partial z}\right)=0$, at $z=h(r)$ 


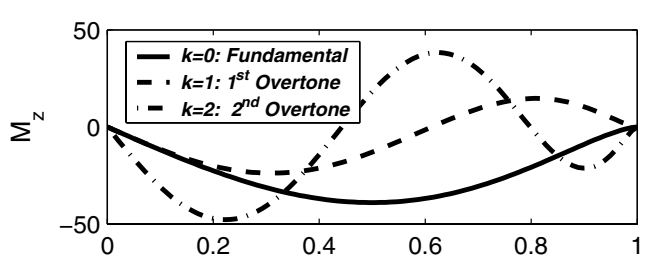

(a) $n=3 / 2$

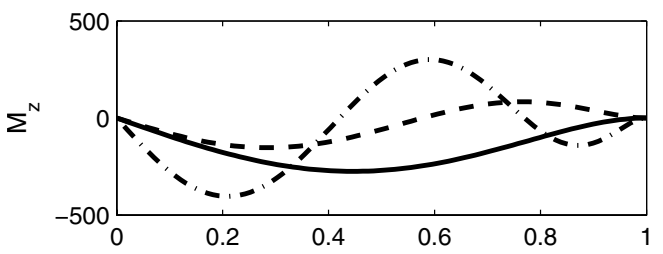

(b) $n=2$
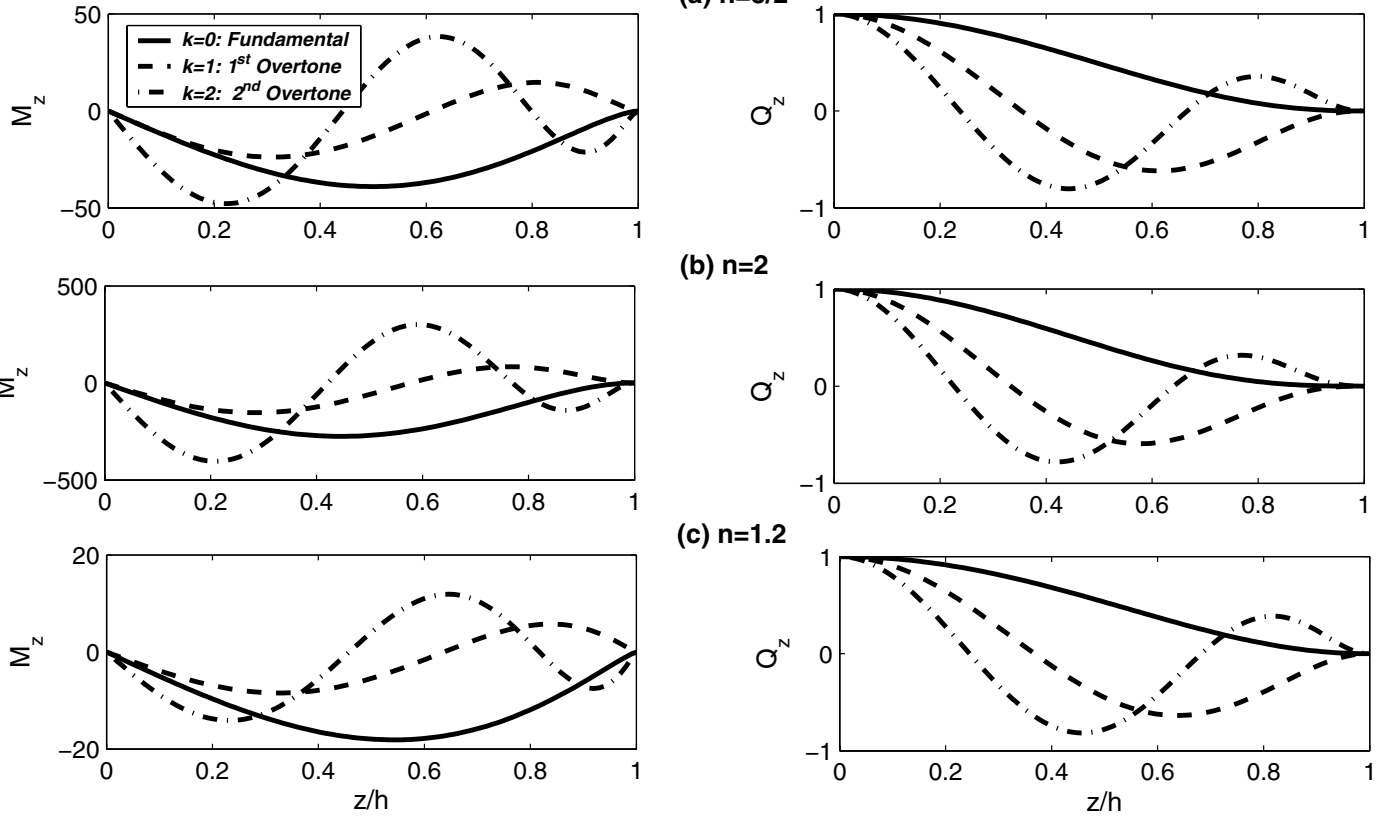

(c) $n=1.2$

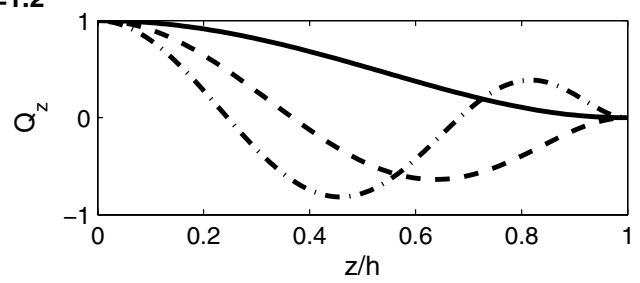

Fig. 1. Structure functions for the homogeneous acoustic modes for a few values of the polytropic index and overtone number for values of $\alpha=0.1$. Plotted are the vertical mass flux $M_{z}=\rho_{0} \hat{v}_{\mathrm{h}}^{\prime}$ and $Q_{z}=P_{0} \partial \hat{v}_{\mathrm{h}}^{\prime} / \partial z$. All $Q_{z}$ are scaled to their values at $z=0$.

which, we must reiterate, is at the unperturbed disk boundary. Also, the stress conditions are

$\eta r \frac{\partial \Omega_{2}^{\prime}}{\partial z}=0$ and $\eta \frac{\partial u_{1}^{\prime}}{\partial z}=0$, at $z=h(r)$.

Finally, the integrated mass flux condition is equivalent to requiring

$2 \pi r \int_{-h}^{h} \rho_{0} u_{1}^{\prime} \mathrm{d} z=0$.

We turn now to the presentation of analytic solutions of the system (38), (39). We shall first discuss, in Sect. 4.2, the case $u_{1}^{\prime}=0$ (and thus also $\Omega_{2}^{\prime}=0$ ), i.e. solving only the homogeneous part of Eq. (39). The solutions of this equation constitute the above mentioned first pair of modes (purely vertical disturbances) and we shall refer to them as vertical acoustics. The solutions to the full set (38), (39) in the general case will be discussed in Sect. 4.3. This pair of modes will be referred to as driven general acoustics. The transient growth behavior found in our solutions will be detailed in Sect. 4.4. A full exposition of the calculations leading to the solutions quoted and discussed in Sects. 4.2-4.4 is presented in Appendices B.1 and B.2. In the next three subsections only the salient features will be given.

\subsection{Results: (i) vertical acoustics}

The general solution to the inhomogeneous Eq. (39) can be written, in the form

$v_{2}^{\prime}=v_{\mathrm{h}}^{\prime}+v_{\mathrm{p}}^{\prime}$

where the index $\mathrm{h}$ stands for a solution of the homogenous equation and the index $\mathrm{p}$ stands for particular solution of the inhomogeneous equation. As we have already remarked, the thinness of the disk means that the density and vertical velocity fluctuations do not induce either radial or azimuthal motions at these lowest orders. This implies that homogeneous solutions of Eq. (39), (i.e. with the equation's rhs set to zero, because $u_{1}^{\prime}=\Omega_{2}^{\prime}=0$ say, see above) are perfectly acceptable.

These solutions have the form (see Appendix B.1)

$v_{\mathrm{h}}^{\prime}=\hat{v}_{\mathrm{h}}^{\prime}(z, r) \exp (\tilde{\sigma} T)+$ c.c. $\quad$ with $T \equiv \frac{t}{r^{3 / 2}}$

where the spatial eigenfunctions $\hat{v}_{\mathrm{h}}^{\prime}$ are composed of the associated Legendre functions. This form of $v_{\mathrm{h}}^{\prime}$ already indicates that these solutions are inseparable in $r$ and $t^{4}$. The temporal eigenvalues, which appear in complex conjugate pairs, are functions of $\alpha$, the polytropic index $n$ and the acoustic overtone (labelled by the integer index $k$, say). Thus the eigenvalues of the vertical acoustic modes should actually be written as $\tilde{\sigma}_{k}(\alpha, n)$. For instance, the eigenfrequency of the fundamental (i.e., $k=0$ ) mode, is given by

$\tilde{\sigma}_{k=0}(\alpha, n) \equiv \tilde{\sigma}_{\mathrm{F}}=-\frac{4}{9} \alpha \pm \mathrm{i}\left|\left(\frac{16}{81} \alpha^{2}-\frac{2 n+1}{n}\right)\right|^{1 / 2}$,

$0<\alpha<1 ; n>1$.

Fundamental modes, as well as all other overtones, show decay (see Appendix B.1). In Fig. 1 we display the spatial structure of the eigenfunctions $\hat{v}_{\mathrm{h}}^{\prime}$ and their vertical gradient $\partial \hat{v}_{\mathrm{h}}^{\prime} / \partial z$ for the first three mode indices and for three polytropic indices. The structure of the eigenfunction is found not to be very sensitive to the polytropic index.

${ }^{4}$ Note that this basic inseparability is tied to the presence of a continuous spectrum. 


\subsection{Results: (ii) driven general acoustics}

We turn to the general solution to Eqs. (38), (39) by first focusing on Eq. (38). This equation admits an infinite set of eigenmode solutions in a way similar in character to the homogeneous solutions discussed before. The general derivation and structure of these eigenmodes is detailed in Appendix B.2. For the sake of clarity, the discussion in the main text here focuses only upon the dynamics associated with the fundamental mode of the operator $\mathcal{P}$ and we shall restrict our attention to the case $n=3 / 2$.

The solution to the dynamical radial velocity profile in an $n=3 / 2$ polytrope is given by,

$u_{1}^{\prime}=\hat{u}^{\prime}(z, r) \mathrm{e}^{\sigma T+\varphi}+$ c.c., with $T=\frac{t}{r^{3 / 2}}$

in which the spatial eigenfunction $\hat{u}^{\prime}(z, r)$ has the particularly simple structure

$\hat{u}^{\prime}=A(r)\left(\frac{z^{2}}{h^{2}}-\frac{1}{6}\right)$.

$A(r)$ is an amplitude whose radial functional form is technically arbitrary and would be set by the initial disturbance condition. An arbitrary phase factor of $\varphi$ is also introduced. The eigenvalues, denoted in this case by $\sigma$, come in complex conjugate pairs given by

$\sigma= \pm \mathrm{i}-\frac{8}{5} \alpha$.

Without loss of generality we need only consider the "+" solution since the phase factor $\varphi$ takes care of the "-" solution. The temporal behavior of these modes is again one of decaying oscillations, but with frequencies given by the local rotation rate of the disk. Note the prescription for $h$ given in Eq. (26): for values of $r$ sufficiently greater than the zero-torque radius, $r_{*}$, the functional dependence of the disk height with respect to $r$ is well approximated by,

$h(r) \sim h_{1} r, \quad \frac{\mathrm{d} h}{\mathrm{~d} r} \sim h_{1}$,

where $h_{1}$ is determined by the mass flux rate and the value of $\alpha$ according to Eq. (27) or, for more general values of $n$, by Eqs. (A.4) and (A.6). Since $h_{1}$ simply adds a multiplicative factor to all the dynamical quantities, it plays no role in the quality of the ensuing evolution and, as such, we set $h_{1}=1$ without any loss of general flavor.

With this solution for $u_{1}^{\prime}$ we may find a particular solution to the vertical velocity, $v_{\mathrm{p}}^{\prime}$ by solving Eq. (39) directly. The details of this procedure are presented in Appendix B.2 but we highlight the major steps here.

We posit the following form for $v_{\mathrm{p}}^{\prime}$ and $\rho_{\mathrm{p}}^{\prime}$,

$v_{\mathrm{p}}^{\prime}=\hat{v}_{\mathrm{p}}(\zeta, r) \mathrm{e}^{\sigma T}+\hat{V}_{\mathrm{p}}(\zeta, r)\left(T \mathrm{e}^{\sigma T}\right)+$ c.c.,

$\rho_{\mathrm{p}}^{\prime}=\hat{\rho}_{\mathrm{p}}(\zeta, r) \mathrm{e}^{\sigma T}+\hat{R}_{\mathrm{p}}(\zeta, r)\left(T \mathrm{e}^{\sigma T}\right)+$ c.c.,

where $\zeta \equiv z / h(r)$. Recall that we solve only for the fundamental mode of the system (again see Appendix B.2) and that there is obviously a dependence on $\alpha$. It is important to notice the explicit appearance of a multiplicative $T$ term in the above expression. This temporal functional dependence is necessary in order to balance terms arising on the rhs of Eq. (39). In general, the form of these lowest order structure eigenfunctions is

$\left(\begin{array}{c}\hat{v}_{\mathrm{p}} \\ \hat{V}_{\mathrm{p}}\end{array}\right)=\left(\begin{array}{c}a_{1} \\ b_{1}\end{array}\right) \zeta+\left(\begin{array}{l}a_{3} \\ b_{3}\end{array}\right) \zeta^{3}$,

namely, it is polynomial in $\zeta$ of the third degree with only odd powers in $\zeta$. The four coefficients are functions of $r$ and $\alpha$, i.e. $a_{i}=a_{i}(r, \alpha)$ and $b_{i}=b_{i}(r, \alpha)$ for $i=1,3$.

The solution to the density perturbation, $\rho_{\mathrm{p}}^{\prime}$ is straightforwardly obtained using Eqs. (51), (53) along with $\rho_{0}$ defined by Eq. (19) and then integrating Eq. (36) with respect to time. This gives

$\left(\begin{array}{l}\hat{\rho}_{\mathrm{p}} \\ \hat{R}_{\mathrm{p}}\end{array}\right)=\left(1-\zeta^{2}\right)^{\frac{1}{2}}\left[\left(\begin{array}{c}c_{0} \\ d_{0}\end{array}\right)+\left(\begin{array}{c}c_{2} \\ d_{2}\end{array}\right) \zeta^{2}+\left(\begin{array}{c}c_{4} \\ d_{4}\end{array}\right) \zeta^{4}\right]$,

where, again, the coefficients are functions of $r$ and $\alpha$, that is, $c_{i}=c_{i}(r, \alpha)$ and $d_{i}=d_{i}(r, \alpha)$ for $i=0,2,4$.

The polynomials appearing in the square brackets of the expressions for $\hat{\rho}_{\mathrm{p}}$ and $\hat{R}_{\mathrm{p}}$ have only even powers of $\zeta$. The detailed forms of the coefficients depend on the form of the generalized initial perturbation radial structure $A(r)$ as well as on $n$. We avoid presenting them here because they are very long and cumbersome; and the details of the coefficients have no effect on the resulting transient dynamics since they describe only a particular type of disturbance. For the sake of simplicity all of the analytic results which will be presented below assume $A=\mathrm{e}^{\mathrm{i} \pi / 4}$ (that is, one particular form of the initial conditions of the radial disturbance) and $n=3 / 2$.

\subsection{Results: (iii) transient dynamics and growth}

As is evident by inspection of Eq. (53), $v_{\mathrm{p}}^{\prime}$ and $\rho_{\mathrm{p}}^{\prime}$ exhibit a pronounced transiently growing phase before eventually decaying. To better demonstrate this more clearly we consider the integral quantities

$\mathcal{E}_{\mathrm{a}}(r, t ; \alpha) \equiv h(r) \int_{-1}^{1}\left(\frac{1}{2} \rho_{0} v_{\mathrm{p}}^{\prime 2}+\frac{1}{2} \frac{c_{\mathrm{s} 0}^{2} \rho_{\mathrm{p}}^{\prime 2}}{\rho_{0}}\right) \mathrm{d} \zeta$,
$E_{\mathrm{a}}(t ; \alpha) \equiv \int_{r_{\text {min }}}^{r_{\max }} \mathcal{E}_{\mathrm{a}} 2 \pi r \mathrm{~d} r$.

The dynamical quantity $\mathcal{E}_{\mathrm{a}}(r, t ; \alpha)$ is to be interpreted as the acoustic energy (per unit area of the disk) consisting of the kinetic energy in the vertical velocity disturbances and the compression energy due to the density disturbances (see Sect. 5). The contribution of the (purely oscillatory) velocity components, resulting from the homogeneous part the disturbance equations, are left out when forming this energy integral. As $\mathcal{E}_{\mathrm{a}}$ is a function of radial position $r$, one may form the quantity $E_{\mathrm{a}}(t ; \alpha)$, i.e., the total disturbance acoustic energy (in this sense) of the disk, by integrating over the radial range in which these disturbances were assumed to exist, that is, between the inner and outer bounds $r_{\min }$ (significantly above the inner zero-torque radius) and $r_{\max }$ (significantly below the outer disk edge). 


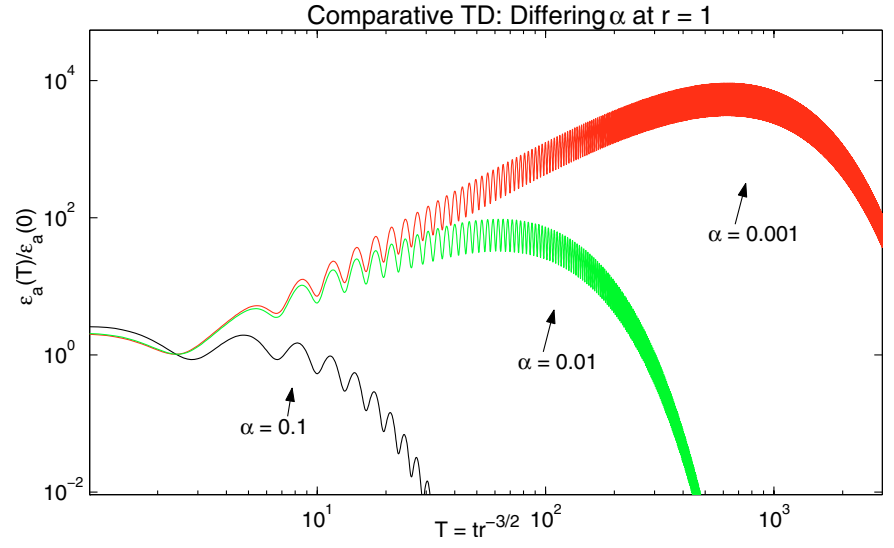

Fig. 2. The transient growth in the quantity $\mathcal{E}_{\mathrm{a}}$ for the fundamental mode at $r=1, n=3 / 2$ and where $A(r)=\mathrm{e}^{\mathrm{i} \pi / 4}, \mathrm{~d} A / \mathrm{d} r=0$. The four curves correspond to $\alpha=0.1,0.01,0.001$ and are presented on a log$\log$ plot. All curves are $\mathcal{E}_{\mathrm{a}}$ scaled to its corresponding value at $T=0$. The general rise time is proportional to the inverse of $\alpha$ as well as the maximum amplitude achieved. $\mathcal{E}_{\mathrm{a}}$ is also characterized by oscillations with period $\pi$ that sit atop the general transient trend.

In general, both $\mathcal{E}_{\mathrm{a}}$ and $E_{\mathrm{a}}$ are functions of the $\alpha$ parameter as well as the structure of the radial velocity perturbation $A(r)$ (see Eq. (51)). As we have assumed (see Sect. 4.3) $A(r)$ is taken here to be constant with respect to $r$, making the integrals defined in Eq. (57) analytically tractable. Nonetheless the expressions, which have been verified with the aid of Mathematica 5.0, are very long and are not displayed here. Only their essential features, in particular their dependence upon $\alpha$ and $r_{\max }$, are addressed here.

With $A=\mathrm{e}^{\mathrm{i} \pi / 4}$ we find that $\mathcal{E}_{\mathrm{a}}$ has the functional form

$\mathcal{E}_{\mathrm{a}}(r, t ; \alpha)=\frac{\mathrm{e}^{-\alpha 2 T}}{r^{3 / 2}} \Upsilon(T, \cos 2 T, \sin 2 T ; \alpha)$,

where $\Upsilon$ is a well-defined analytical, albeit complicated, function of its arguments. Thus, $\mathcal{E}_{\mathrm{a}}$ depends on time only through the variable $T=t / r^{3 / 2}$, which is the time measured in units of the local disk rotation period, at $r$, divided by $2 \pi$. Different values of $r$ merely change the overall amplitude of the response as governed by the coefficient multiplying the function $\Upsilon$. Otherwise, the evolution of $\mathcal{E}_{\mathrm{a}}$ in the disk is self similar and is always decaying at long times $T$. Because of this we display below the evolution of $\mathcal{E}_{\mathrm{a}}$ for different values of $\alpha$ at $r=1$ only.

Inspection of Fig. 2, which plots against $T$ the value of $\mathcal{E}_{\mathrm{a}}(T) / \mathcal{E}_{\mathrm{a}}(0)$ for a range of $\alpha$ values, uncovers a very important property of the transient growth. As $\alpha$ decreases the magnitude of the growth becomes more prominent (for example, it is up to $\sim 3$ orders of magnitude for $\alpha=0.001$ ) and the maximum occurs at correspondingly later times. The time corresponding to the maximum amplitude $T_{\max }$ is roughly $\sim 5 /(8 \alpha)$ in this ${ }^{5}$ case. The rise in energy is modulated by oscillations which arise from the fact that there are correlations between pairs of variables which contribute to the overall growth in $\mathcal{E}_{\mathrm{a}}(T)$ (see Sect. 5). These variables oscillate in a frequency defined by

\footnotetext{
${ }^{5}$ For the $k$ th overtone, $T_{\max }$ will be smaller by a factor of $1 / k^{2}$, see Eq. (B.12).
}

the imaginary part of the eigenvalue (52) and because $\mathcal{E}_{\mathrm{a}}(T)$ is composed of products of pairs of dynamical variables terms depending on $2 T$ appear - explaining why the observed period is half the orbital period at a given radius.

Figure 3 depicts the behavior of $E_{\mathrm{a}}$ for assorted values of $\alpha$ and $r_{\max }$. In all the cases displayed we have assumed that the inner radius, $r_{\min }$, to be 0.05 . For given $r_{\max }$, smaller values of $\alpha$ correspond to more dramatic instances of growth before final decay. The peak magnitudes are proportional to the inverse of $\alpha$ as is also the time when the peak occurs - a trend which is similar to that observed in the quantity $\mathcal{E}_{\mathrm{a}}$. It is very important to keep in mind that $A(r)$ affects the spatial details but not the global time behavior. Finally, we point out that the occurrence of the peak energy amplitude is delayed for larger values of $r_{\max }$, at given values of $\alpha$, although the value of the energy at the peak time remains roughly the same.

To get a sense for the expected qualitative spatio-temporal behavior of such transiently growing solutions we have computed the results for a case in which $A(r)$ is not constant (and, thus, the possibility to evaluate the relevant integrals analytically can not be expected in general). We consider here an initial condition in which the radial velocity amplitude has a Gaussian form centered around some fixed radius,

$A(r)=\mathrm{e}^{\mathrm{i} \pi / 4} \mathrm{e}^{-\frac{\left(r-r_{0}\right)^{2}}{\Delta_{f}}}$.

In our particular case we take the full width at half maximum given by $\Delta_{f}=2.5$ and the initial center of the disturbance $r_{0}=3$. Other parameters are $n=3 / 2$ and $\alpha=0.01$ and $\epsilon=0.05$. The fluid is otherwise undisturbed. As pointed out above, with this form for $A$ we loose the ability to find an analytic solution and we must resort to numerical evaluation. In Fig. 4 we show, in a contour plot in the disk meridional plane, the kinetic energy (per unit volume) contained in the vertical velocity disturbance $v_{\mathrm{p}}$, that is, $\rho_{0} v_{\mathrm{p}}^{2} / 2$. The transient growth and decay of the disturbance is shown in the time sequence of figures displaying the spatial structure of the disturbance. We also depict how the disk surface moves in response to the imposed perturbation by solving the equation of motion for the boundary at this order. In the figure time is quoted in terms of rotation times of the disk as measured at the radius $r=1$. Because the response of the disk surface is an integral with respect to time, it is not a surprise to see a large amplitude in the surface position long after the kinetic energy has died away. The two dramatic things to note is that, (a) there maximal rise in the disturbance energy is of almost three orders of magnitude and (b) the physical amplitude of fluctuations in the disk surface cease, from a certain time and on, to be very small. Associated with the last effect is the emergence, as time moves on (by $t \sim 2000$ ), of crenellated patterns on the disk surface. This crenellation is a result of the epicyclic frequencies causing the amplitude profile to wind-up on itself. Eventually, the winding is so strong that viscous effects take over and wipe the perturbations away by a several thousand rotation times. Too high an amplitude and too short a wavelength of the crenellation may give rise to the appearance of terms and gradients that formally can "break order" in our asymptotic solution, but the validity of the main result (the very substantial transient growth 

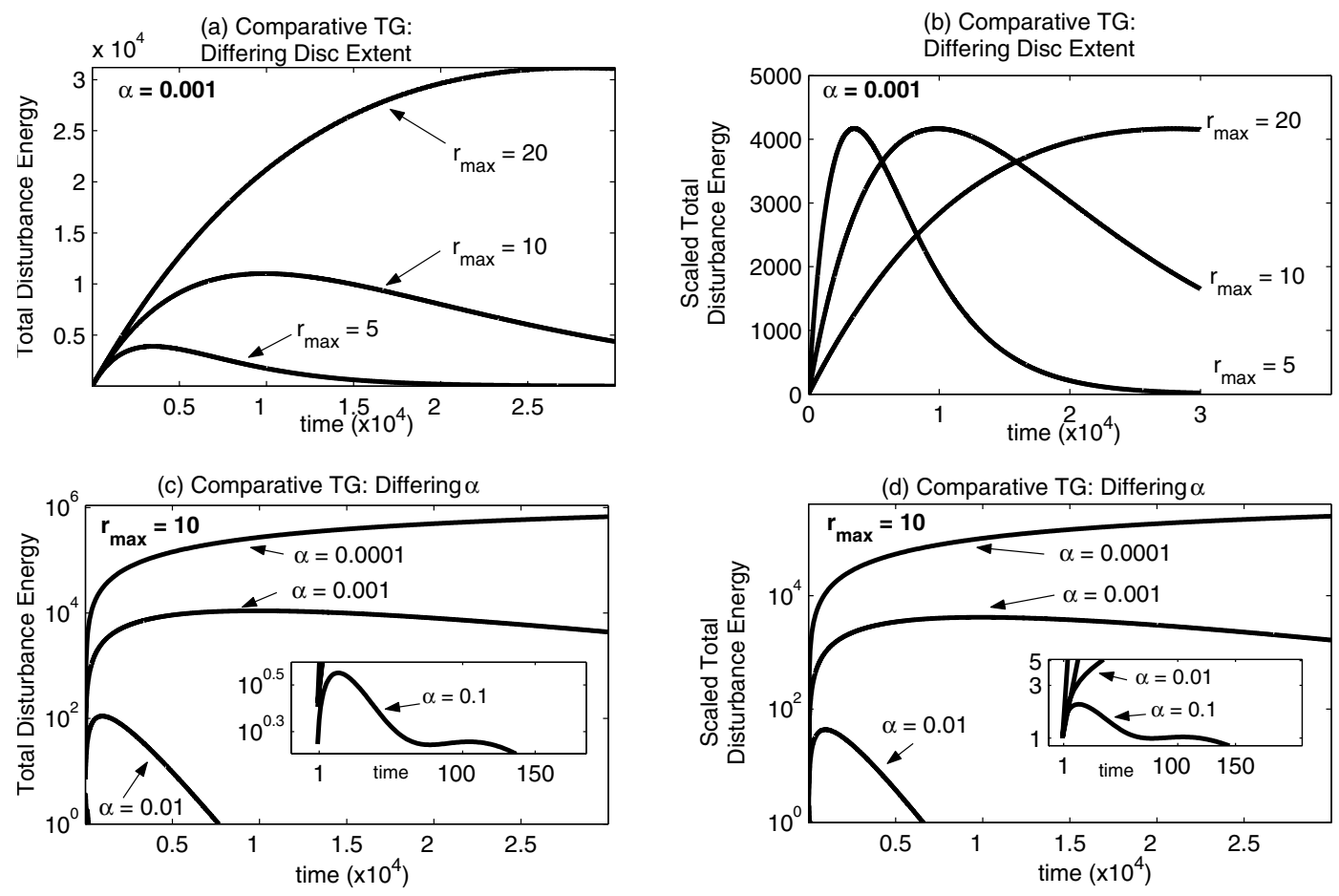

Fig. 3. Comparative transient behavior of the "total disturbance energy" $\left(E_{\mathrm{a}}\right)$ and "scaled disturbance energy" $\left(E_{\mathrm{a}}^{(\mathrm{s})} \equiv E_{\mathrm{a}}(t) / E_{\mathrm{a}}(0)\right)$. In all plots $n=3 / 2, r_{\min }=0.05$ and $A(r)=\mathrm{e}^{\mathrm{i} \pi / 4}$. Panel a) depicts $E_{\mathrm{a}}$ for different values of $r_{\max }$ with $\alpha=0.001$. Larger values of $r_{\max }$ simply correspond to longer lived phenomena before decay ultimately sets in at larger values of the disk radius meaning that the total energies are larger. In panel b) $E_{\mathrm{a}}^{(\mathrm{s})}$ is shown. $E_{\mathrm{a}}^{(\mathrm{s})}$ achieves the same overall maximum amplitude before decay. Similarly panels c) and d) depict $E_{\mathrm{a}}$ and $E_{\mathrm{a}}^{(\mathrm{s})}$ for $\alpha=0.1,0.01,0.001$ at $r_{\max }=10$. The trend for the $E_{\mathrm{a}}$ to grow with decreasing $\alpha$ is evident. In d) we see that this growth, in terms of scaled energies, can be dramatic - by nearly three orders of magnitude.

in the disturbance energy) is quite robust and may be justified by more sophisticated asymptotic treatment (see Sect. 5). In any case, for $\alpha$ not too small and time not too large there is no reason for concern in this respect.

\subsection{The $\alpha \rightarrow 0$ limit: algebraic growth}

The limit of zero viscosity, i.e. $\alpha \rightarrow 0$, results in unbounded algebraic growth. For $n=3 / 2$, an arbitrary initial amplitude $A(r)$ and the unapproximated disk height $h(r)$, the vertical velocity fluctuation $v_{\mathrm{p}}^{\prime}$ simplifies to

$$
\begin{aligned}
\lim _{\alpha \rightarrow 0} v_{\mathrm{p}}^{\prime}= & \mathrm{e}^{\mathrm{i} T} \frac{A h}{r}\left(\frac{6}{7} \frac{r}{h} \frac{\mathrm{d} h}{\mathrm{~d} r}-\frac{1711}{1470}+\frac{5}{21} \frac{r}{A} \frac{\mathrm{d} A}{\mathrm{~d} r}-T \frac{5 \mathrm{i}}{14}\right) \zeta \\
& +\mathrm{e}^{\mathrm{i} T} \frac{A h}{r}\left(\frac{8}{21} \frac{r}{h} \frac{\mathrm{d} h}{\mathrm{~d} r}+\frac{110}{147}-\frac{4}{21} \frac{r}{A} \frac{\mathrm{d} A}{\mathrm{~d} r}+T \frac{2 \mathrm{i}}{7}\right) \zeta^{3} .
\end{aligned}
$$

The density follows in similar suit. Notice that though the viscous effects vanish, the oscillation frequency associated with the local epicyclic vibrations remain implying that solutions in this limit appear as algebraically growing oscillations. This result is consistent with the more general (than that with the polytropic assumption) analysis made for disks with in which the fluctuations are adiabatic (Umurhan \& Shaviv 2005).

It is also important to note that the limiting process of $\alpha \rightarrow 0$ is one that cannot be taken without caution and care. This is because this is a singular limit - substituting simply $\alpha=0$ gives rise to a singularity in the expression for the steady state height $h(r)$. Inspection of the solution to $h(r)$ as appearing in Eq. (A.5) shows that $h$ diverges as $\alpha \rightarrow 0$. However, one could formally take this limit holding the ratio $\dot{M} / \alpha$ fixed (cf. Eq. (A.5) and the definition of $\Lambda$ in Eq. (A.4)), namely

$0<\lim _{\alpha \rightarrow 0} \frac{\dot{M}}{\alpha}<\infty$,

because the vanishing of the viscosity coefficient gives rise to a vanishing mass transfer rate.

\section{Summary and discussion}

This paper is a contribution to the investigation of the purely hydrodynamic response of thin, viscous, axisymmetric accretion disks to initial perturbations. Almost all of the recent studies, in which the phenomenon of transient growth has been found in accretion disks, were based on local (using the "shearing box" approximation) analysis (see references cited in Sect. 1) of an inviscid (or almost inviscid) shear flows. By contrast, we have considered an analysis of the dynamics of a substantially large global section of a viscous accretion disk. To our knowledge the most recent work in a similar direction is the fully numerical study by Klahr \& Bodenheimer (2003). In an endeavor to investigate disturbances which are not limited to the shearing box (on which, typically, periodic boundary conditions are imposed), but still be able to treat the problem analytically or semi-analytically, we have resorted to asymptotic methods. Adopting such a method, previously applied (in $\mathrm{R}$ and $\mathrm{KK}$ ) to steady thin (i.e. $\epsilon \ll 1$ ) axisymmetric viscous disks 


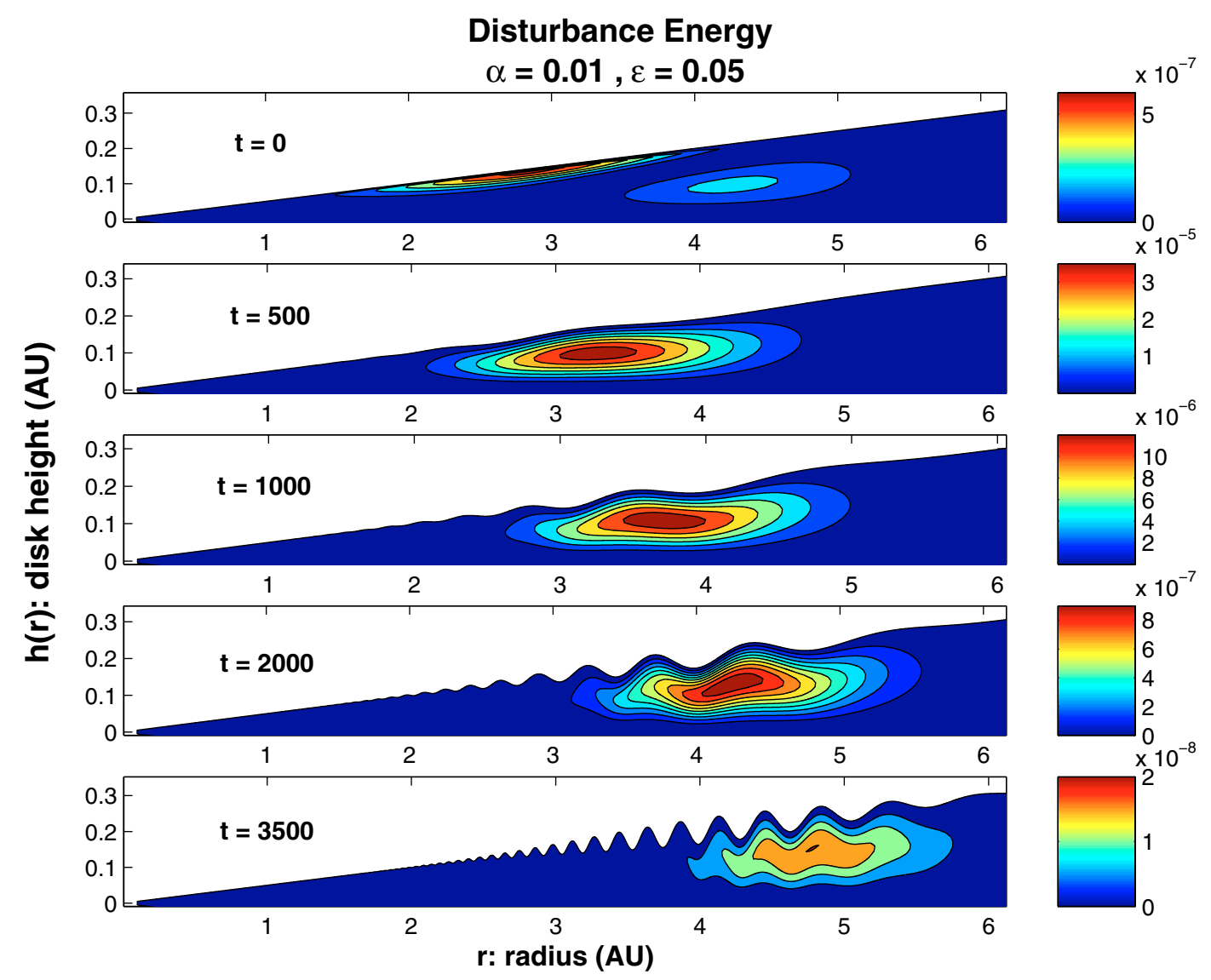

Fig. 4. Transient growth in the disturbance kinetic energy density in a disk where $n=3 / 2, \alpha=0.01, \epsilon=0.05$ and $A(r)=\exp i \pi / 4 \times$ $\exp \left(r-r_{0}\right)^{2} / \Delta_{\mathrm{f}}$, with, in this example, $r_{0}=3$ and the full width-half-maximum $\Delta_{\mathrm{f}}=2.5$. Time is quoted in terms of disk rotation times at the radius $r=1$ so that $T=t$. The magnitude of the disturbance energy density is indicated by the various colors (whose meaning is indicated on the right of each panel) on a cut through the disk's meridional plane. Significant growth in the disturbance energy is observed (almost three orders of magnitude). Strong crenellation patterns appear in the fluctuating boundary of the disk before ultimate viscous decay takes over and wipes the pattern out.

and extending it to include time-dependence, we are able to show that initial disturbances give rise to transient dynamics, in which substantial transient growth emerges on the global scale on the disk. Moreover, we have found analytical expressions that help in understanding the underlying physics giving rise to the transient growth (see below). In achieving this goal a number of simplifying assumptions have been made. The unperturbed steady disk and the disturbances were assumed to be polytropic and axisymmetric and they were treated in a ring whose inner (outer) limit was significantly distant from the inner (outer) physical disk boundary. In addition, we chose to use the simplest initial conditions so as to excite the most fundamental modes, which we could readily analyze. Note, however, that our approach addresses directly the initial value problem, that is it is non-modal (see e.g. Criminale et al. 2003; Schmid \& Henningson 2001)

As pointed out before, the simplifying assumptions have largely been made to allow for analytical treatment, which is able to bring out, and allow for an analysis of, some salient features of the problem. In this framework we were able to derive a set of dynamic equations for successive orders of $\epsilon$. We stopped at the $\epsilon^{2}$ order, but the expansion can be carried out to higher order terms. We were able to identify two kinds of response and obtained the analytical structure of some simple initial disturbances. To $O\left(\epsilon^{2}\right)$ we have determined an analytical solution to the dynamic problem and discovered that this set of equations manifests strong transient growth, irrespective of the polytropic index $n$. As we have noted, the severity of the transient growth is inversely proportional to $\alpha$ so that as the viscosity gets small, the transient growth becomes more dramatic. It seems then that transient growth is an intrinsic property of global axisymmetric disturbances of polytropic disks and, given the results of previous works done in the local "shearing box" approximation as well as the two-dimensional (in the disk plane) global case (Ioannaou \& Kakouris 2002), we may reasonably expect it to be a property of all accretion disks.

In spite of the simplifying assumptions we have implemented, and whose relaxation must be addressed in the future (see below), it is important to remark that the asymptotic expansion is not equivalent to the customary (in linear stability analysis) linearization procedure of infinitesimal perturbations imposed on a global steady state. Although, to the orders to which solutions have been determined the governing equations are linear, this procedure is intrinsically a finite-amplitude expansion (see Umurhan \& Shaviv 2005). Indeed, technically speaking, at each $\epsilon^{n}$ order, the equations are linear with 
inhomogeneous terms that result from the $\epsilon^{n-1}$ order. Yet the aggregate solution, and the region of its validity, put it in the finite-amplitude realm.

We turn now to the investigation of the physical sources for the transient growth (TG) that we have found in what we have called "general driven acoustic modes". To this end we consider the following energy density function

$\varepsilon_{\mathrm{a}}(r, z, t) \equiv \frac{1}{2} \rho_{0}{v_{2}^{\prime}}^{2}+\frac{1}{2} \frac{c_{\mathrm{s} 0}^{2}}{\rho_{0}} \rho_{2}^{\prime 2}$.

The first term in this expression represents the kinetic energy contained in the time dependent part of vertical motion while the second term represents a sort of thermal energy in the form of an acoustic disturbance (Rayleigh 1877). We have already considered integrals of a similar function, but only for the particular solution $v_{\mathrm{p}}^{\prime}$ (see Sect. 4.4). Here we shall be interested in the temporal behavior of the volume integral of $\varepsilon_{\mathrm{a}}$ over the entire domain of interest $r_{\text {in }} \leq r \leq r_{\text {out }} ;|z| \leq h$.

Using Eqs. (35), (36) to substitute for the relevant timederivatives and converting the resulting integrals to surface integrals whenever possible in the usual way, we get

$$
\begin{aligned}
\frac{\mathrm{d}}{\mathrm{d} t} \int_{V} \varepsilon_{\mathrm{a}} \mathrm{d} V= & -\int_{S}\left(\rho_{2}^{\prime} v_{2}^{\prime} c_{\mathrm{s} 0}^{2}\right) \mathrm{d} S+\frac{4}{3} \int_{S}\left(\eta v_{2}^{\prime} \frac{\partial v_{2}^{\prime}}{\partial z}\right) \mathrm{d} S \\
& -\frac{4}{3} \int_{V} \eta\left(\frac{\partial v_{2}^{\prime}}{\partial z}\right)^{2} \mathrm{~d} S+T_{E},
\end{aligned}
$$

where $T_{E}$ represents volume integrals over the terms that are responsible for transient energy growth and its explicit form is

$$
\begin{aligned}
T_{E}= & \int_{V}\left\{\frac{v_{2}^{\prime}}{r} \frac{\partial}{\partial r}\left(\eta r \frac{\partial u_{1}^{\prime}}{\partial z}\right)\right. \\
& \left.-\frac{2}{3} v_{2}^{\prime} \frac{\partial}{\partial z}\left[\frac{\eta}{r} \frac{\partial\left(r u_{2}^{\prime}\right)}{\partial r}\right]-\frac{\rho_{2}^{\prime}}{\rho_{0}} \frac{c_{\mathrm{s} 0}^{2}}{r} \frac{\partial\left(r \rho_{0} u_{1}^{\prime}\right)}{\partial r}\right\} \mathrm{d} V .
\end{aligned}
$$

The first term on the rhs of Eq. (63) represents the mechanical work done on the disk by the bounding surface $S$. But, given that (a) the pressure of the moving boundary is zero and (b) the quantities in the integrand are odd functions of $z$, this surface integral vanishes. The second term represents the work performed on the surface by the viscous stresses associated with gradients of the vertical velocities. In a similar fashion the surface integral of this term also vanishes. Its general nature is not immediately obvious, but given the solutions we have obtained in this paper we always find that it vanishes, mainly because of the fact that $\eta$ tends to zero (when approaching the disk surface) sufficiently fast. The third term is the volume integrated loss of energy due to viscous dissipation, i.e. the viscous losses. Since the integrand here is positive definite, this term is always a sink of energy and is the ultimate cause of decay in all the perturbations. Thus we concisely and transparently write the last relation as,

$\frac{\mathrm{d}}{\mathrm{d} t} \int_{V} \varepsilon_{\mathrm{a}} \mathrm{d} V=T_{E}-$ [viscous losses].

Consider now the structure of the expression for $T_{E}$ in more detail. The first two terms in Eq. (64) are explicitly viscosity dependent, while the third is not, although it may depend on $\alpha$ implicitly, via the $\alpha$ dependence of the disturbances. The sign of these terms may fluctuate in time, and in principle they are of the same order as the other terms. Ultimately all activity decays and therefore there is no possibility of a viscous instability. The first two terms consist of the cross-correlations between the vertical and horizontal viscous shear disturbances on one hand and the vertical velocity disturbance on the other hand. Regarding the third term, we notice that it follows from Eq. (37) that $\rho^{\prime} c_{\mathrm{s} 0}^{2} / \rho_{0}$ is proportional to the dynamical disturbance of the sound speed, i.e., $c_{\mathrm{s} 2}^{2}$ (or, equivalently, of the temperature), so that the expression in the integrand may be rewritten as

$c_{\mathrm{s} 2}^{2} \frac{1}{r} \frac{\partial\left(r \rho_{0} u_{1}^{\prime}\right)}{\partial r}$

The volume integral of this expression thus depends on the correlation between the dynamical fluctuations in the radial mass flux and its radial derivative with the disturbances in the sound speed. Although we have chosen only disturbances which do not contribute at all to the total horizontal mass transfer rate (see the condition expressed in Eq. (46)), the correlation integral consisting of the third term obviously need not vanish. In other words, the integral condition (46) on the radial velocity dynamical disturbance in the radial velocity does not imply that the contribution to the work done on the fluid, as given in the third term, is zero.

To assess the relative contribution of the first two viscous terms to above discussed third correlation integral, we calculated the relevant ratio and found that for the three values of $\alpha$ displayed in Fig. 2, this ratio decreases monotonously with $\alpha$. For example, for $\alpha=0.001$, it is less than $0.1 \%$ for all $T>0$. Thus, for $\alpha \rightarrow 0$ the TG is totally dominated by the correlations embodied in the third term. This means that the algebraic growth found in the inviscid limit by Umurhan \& Shaviv (2005) must be driven by this term.

We conclude with some remarks on possible improvements to the asymptotic analysis of the sort done here and prospects for the future. Asymptotic expansions, when viable, are often very robust and provide a good approximation to the solution when truncation to only few leading terms is done. Obviously, when a term in the series becomes very large it may "break its order", that is, become of the order of a previous term and as such make the expansion invalid in this region (see next paragraph). In our expansions successive terms ratios are of $O\left(\epsilon^{2}\right)$, and thus the procedure's validity should not severely be limited even up to a growth factor of $\sim 100$ or so (in the velocity or density perturbations).

For instance, the algebraic growth term in the expression for $c_{\mathrm{p}}^{\prime}$ (i.e. $\rho_{\mathrm{p}}^{\prime}$, cf. Eq. (54)) is such that it can break order or even cause an overall breakdown in the expansion procedure (e.g. involving the $O\left(\epsilon^{4}\right)$ radial pressure gradient terms) if the viscous decay is weak and when $T$ becomes $O\left(\epsilon^{-2}\right)$. This would mean that the validity with respect to time for weakly viscous solutions are somewhat proscribed by the thinness of the disk: smaller values of $\epsilon$ mean that the solutions are valid for longer times after the initial disturbance. More importantly, for a given value of $\epsilon$ one must not be too zealous or overreaching by 
attempting to infer the qualitative behavior of the disk for arbitrarily small values of $\alpha$-which, as one will recall, is formally assumed to be an order 1 quantity.

Despite these caveats, the procedure when carried to higher order introduces corrections which are technically non-linear. Careful consideration must be undertaken in order to handle the response at these higher orders. This may entail treating the disturbance amplitudes for the lower order solutions (like $A(r)$ in $\left.u_{1}^{\prime}\right)$ as weakly non-linear governed by a second "slow" time (e.g. the amplitude is instead written as $A=A(r, \tau)$ where $\left.\tau=\epsilon^{2} t\right)$ in a manner analogous to the treatment of non-linear thick polytropes (for example Balmforth \& Spiegel 1996).

The approach used here may be generalized in a number of additional directions. Allowing for non-axisymmetric perturbations, including the disk inner and outer boundary in some kind of boundary layer analysis and relaxing the polytropic assumption seem to be the most obvious generalizations. In such future studies the implications of the presence of backflow in the steady state should be examined as well. In any case, we have found the presence of TG in the simplest cases. It is difficult to imagine that it will be suppressed in the more general conditions although the effect of radiative energy losses on TG must be carefully examined.

The question concerning the ultimate fate of the transiently grown perturbations and their ability to induce a sustained turbulent state (and concomitant angular momentum transport) in the disk remains largely open. In this context it is worthwhile to notice that since the TG decay times are of the order of hundreds of rotation periods, it is conceivable that accretion disks, which are usually not isolated systems, may experience recurrent external perturbations on such time scales and in this way the dynamical activity may be sustained. It is interesting to note that the most severe TG occurs for small $\alpha$ and it is quenched when $\alpha$ becomes large. Thus, if the transient dynamics uncovered here can indeed give rise to a turbulent state (i.e. one in which an effective, rather large $\alpha$ emerges), it is tempting to speculate that this may lead to some kind of self-sustained process (possibly by means of secondary flows) and, thus, inducing turbulent viscosity in an accretion disk.

Extensive numerical calculations of as realistic as possible accretion disks are however needed to decide if transient dynamics induced by initial perturbations per se may lead, through non-linear processes, to sustained turbulence. Such extremely high-resolution global three-dimensional calculations seem, however, to still be well-above (see Lesur \& Longaretti 2005) the ability of the present computer power and it may be advantageous to also exploit sophisticated non-linear asymptotic methods to complement and guide them.

Acknowledgements. We thank P.-Y. Longaretti for providing us with a preprint of his paper with G. Lesur and for his thoughtful comments on this work.

\section{Appendix A: Disk flow solutions for arbitrary polytropic index}

We present here, for the sake of completeness, the steady-state solution, which can be found in KK only for a particular value of the polytropic index.

\section{A.1. Radial velocity solution and the disk height as function of $r$}

One begins by combining Eqs. (22), (23) into a single equation for $u_{1}$. After a little manipulation and upon using Eq. (20) we find:

$$
\begin{gathered}
\Omega_{0}^{2}\left\{\alpha^{2}\left[\frac{\left(h^{2}-z^{2}\right)}{2(n+1)} \frac{\partial^{2}}{\partial z^{2}}-z \frac{\partial}{\partial z}\right]^{2}+1\right\} u_{1}= \\
-\frac{2 \Omega_{0}}{r^{2} \rho_{0}} \frac{\partial}{\partial r}\left(\eta r^{3} \frac{\partial \Omega_{0}}{\partial r}\right)
\end{gathered}
$$

where $h(r)$ is the (still unknown) disk height. The solution to Eq. (A.1) is

$$
\begin{aligned}
& u_{1}(r, \zeta)=\frac{\alpha h^{2}}{r^{5 / 2}} \\
& \quad \times\left\{\frac{(3 n+1)\left[9(n+1)\left(1-\zeta^{2}\right)+8(2 n+3) \alpha^{2}\right]}{9(n+1)^{2}+4(2 n+3)^{2} \alpha^{2}}-\frac{2 r}{h} \frac{\mathrm{d} h}{\mathrm{~d} r}\right\}
\end{aligned}
$$

where $\zeta=z / h$.

We are now in a position to determine the disk height as a function of the radius. First, we see that vertically integrating the continuity Eq. (24), after multiplying it by $2 \pi r$, leads to a constraint whose physical meaning is that the mass flowing through the disk is independent on $r$. In particular, we are left with

$2 \pi \int_{-h}^{h} \mathrm{~d} z \frac{\partial}{\partial r}\left(r \rho_{0} u_{1}\right)=0$,

because it is supposed a priori that the quantity $\rho_{0} v_{2}$ indeed vanishes as $z \rightarrow \pm h(r)$. The satisfaction of Eq. (A.3) is equivalent to saying that

$\dot{M}_{1}=$ const. $=-2 \pi \int_{-h}^{h} \mathrm{~d} z\left(r \rho_{0} u_{1}\right)$,

where $\dot{M}_{1}$ is the mass accretion rate, which is one of the fundamental free parameters in this system ${ }^{6}$.

The next step is the evaluation of the integral in the above equation using Eq. (A.2). It leads to the following first order non-linear ODE for $h(r)$

$$
\begin{aligned}
& \frac{h^{2 n+3}}{r^{3 n+3 / 2}}\left(1-\frac{2 n+3}{3 n+1} \cdot \frac{r}{h} \frac{\mathrm{d} h}{\mathrm{~d} r}\right)= \\
& \Lambda \equiv \frac{\dot{M}_{1}}{\alpha} \frac{\Gamma(n+5 / 2)}{\Gamma(n+1)} \cdot \frac{(2 n+2)^{n}}{\pi^{3 / 2}(3 n+1)} .
\end{aligned}
$$

Though the above is non-linear, its solution is remarkably easy to write down and reads

$h(r)=(2 \Lambda)^{\frac{1}{2 n+3}} r^{\frac{3 n+1}{2 n+3}}(\sqrt{r}-\sqrt{r})^{\frac{1}{2 n+3}}$.

This solution is shaped so that $r_{*}$ is the radius at which the disk experiences zero torque. At this radius our solution is singular (the thickness of the disk becomes zero and the radial flow

\footnotetext{
${ }^{6}$ Note that the minus sign in the above integral statement reflects the convention that a positive mass accretion rate $\dot{M}_{1}>0$ implies physically that the integrated mass flux must be a negative in order for the material to flow inward.
} 
infinite). For this reason we suppose that $r_{*}$ is always significantly smaller than the fiducial radius $r=1$ and never consider these solutions near the zero torque point ${ }^{7}$ Additionally, at the fiducial radius the height of the disk is given as

$h_{1}=h(1)=(2 \Lambda)^{\frac{1}{2 n+3}}\left(1-\sqrt{r_{*}}\right)^{\frac{1}{2 n+3}}$.

If $h_{1}$ is chosen to be some value, then there is a unique ratio of $\dot{M} / \alpha$ which satisfies this constraint.

Some things to note with regard to the disk thickness function: when $n=3 / 2$ the height of the disk varies linearly with radius for $r \gg r_{*}$; when $n>3 / 2$ the structure of the disk is flared, meaning to say $\frac{\mathrm{d}}{\mathrm{d} r} \frac{h}{r}>0$ as $r$ gets large. The overall "thickness" of the thin disk, in which $h_{1}$ is its measure, is related to the accretion rate according to

$h_{1} \sim \dot{M}_{1}^{\frac{1}{2 n+3}}$.

In the case where $n=3 / 2$, the scale of the disk's thickness is proportional $\dot{M}_{1}^{1 / 6}$ which is consistent with the results obtained by KK.

In conclusion of this part of the Appendix we derive the general condition for the occurrence of backflow (see KK, RG). In other words, we find the highest value of $\alpha$ for which the radial velocity switches sign at some point in the midplane of the disk. This may be determined by setting $\zeta$ to zero in Eq. (A.2) and solving the result for $\alpha$. After making use of Eq. (A.5) we find that $\alpha$ and the position $r$ at which the radial velocity in the midplane is zero are related through the equation

$\frac{2}{3} \alpha-\sqrt{\frac{2 n(n+1)}{(2 n+3)^{2}}\left(1-\frac{3 n+1}{2 n} \sqrt{\frac{r_{*}}{r}}\right)}=0$.

There exists thus a critical value of $\alpha=\alpha_{\infty}$, that is, the one obtained for $r \rightarrow \infty$ in Eq. (A.7) and it is given by

$\alpha_{\infty} \equiv \frac{3}{2} \sqrt{\frac{2 n(n+1)}{(2 n+3)^{2}}}$.

Its significance is such that for values of $\alpha>\alpha_{\infty}$ there is no possibility of backflow in the disk. For values of $0<\alpha<\alpha_{\infty}$ there are places in the disk, which includes the midplane, in which the flow is directed outwards. This flow transition, or stagnation point, bifurcates out of $r=\infty$ at the value of $\alpha=\alpha_{\infty}$. For an $n=3 / 2$ polytrope, $\alpha_{\infty} \sim 0.685$, which is the value predicted by KK. For values of $\alpha<\alpha_{\infty}$ the stagnation point, $r_{\text {stag }}$ occurs at

$\frac{r_{\text {stag }}}{r_{*}}=\frac{81}{4} \frac{(n+1)^{2}(3 n+1)^{2}}{\left[9 n(n+1)-2(2 n+3)^{2} \alpha^{2}\right]^{2}}$,

which, in other words, means to say that for a given value of $\alpha<\alpha_{\infty}$, backflow occurs only for values of $r>r_{\text {stag. }}$. Finally,

${ }^{7}$ None the less, it is clear that the infinities appearing near $r_{*}$ indicate that these solutions breakdown in that vicinity and that a boundary-layer type analysis will be required to cope with the failure. By restricting ourselves to be sufficiently far away from this pathological point we may proceed with the subsequent analysis. there is also a minimum radius $r_{\text {stag }}^{\min }$, below which there is never backflow. This occurs in the limit where $\alpha \rightarrow 0$ and is given by,

$\frac{r_{\text {stag }}^{\min }}{r_{*}}=\left(\frac{3 n+1}{2 n}\right)^{2}$.

In other words it says that $r_{\text {stag }}$ appears in the $\alpha \rightarrow 0$ limit at the value $r_{\text {stag }}^{\min }$. In the case $n=3 / 2$, this absolute minimum stagnation point appears at $(121 / 36) r_{*}$ (see KK).

\section{A.2. $\Omega_{2}, v_{2}$ and $\rho_{2}$}

The general solution to $\Omega_{2}$ comes from inserting $u_{1}$ into Eq. (23) and solving. We find that,

$$
\begin{aligned}
\Omega_{2}= & \frac{h^{2}}{4 r^{7 / 2}}\left[\left(1-\zeta^{2}\right) \frac{2 r}{h} \frac{\mathrm{d} h}{\mathrm{~d} r}\right. \\
& \left.-3\left(1-\frac{4(3 n+1)\left(1-(2 n+3) \zeta^{2}\right) \alpha^{2}}{9(n+1)^{2}+4(2 n+3)^{2} \alpha^{2}}\right)\right] .
\end{aligned}
$$

Notice that the correction $\Omega_{2}$ to the rotational speeds is nonzero in the limit of $\alpha \rightarrow 0$. This merely represents the fact that at this order the pressure contributes something to the overall equilibrium of the system and this contribution makes the flow slightly sub-Keplerian. Notice that unlike $\Omega_{0}$, the solution for $\Omega_{2}$ shows that the angular velocity is no longer constant on cylinders since $\partial \Omega_{2} / \partial \zeta \neq 0$ in general.

Using Eq. (20) for $\rho_{0}$, together with Eq. (A.4), one may straightforwardly, though cumbersomely, integrate Eq. (24) to yield a solution for the vertical velocity. The result is

$$
\begin{aligned}
v_{2}= & \frac{\alpha \zeta h^{3}}{2 r^{7 / 2}}\left[\left(\frac{2 r}{h} \frac{\mathrm{d} h}{\mathrm{~d} r}\right)^{2}-\frac{8(2 n+3)(3 n+1) \alpha^{2}}{9(n+1)^{2}+4(2 n+3)^{2} \alpha^{2}}\left(\frac{2 r}{h} \frac{\mathrm{d} h}{\mathrm{~d} r}\right)\right. \\
& \left.-27 \frac{(n+1)(2 n+1)(3 n+1)\left(1-\zeta^{2}\right)}{(2 n+3)\left(9(n+1)^{2}+4(2 n+3)^{2} \alpha^{2}\right)}\right] .
\end{aligned}
$$

The expression for the density is quite lengthly and we only present it for the case $n=3 / 2$. The integration constants in deriving $\rho_{2}$ were chosen so that $c_{\mathrm{s} 0}^{2} \rho_{2} / \rho_{0} \rightarrow 0$ as $\zeta \rightarrow 0$,

$$
\begin{aligned}
\rho_{2}= & -\frac{\left(1-\zeta^{2}\right)^{3 / 2} h^{5}}{360 \sqrt{5} r^{13 / 2}} \\
& \times\left[3 \frac{675\left(1+\zeta^{2}\right)+16 \alpha^{2}\left(460+528 \alpha^{2}-189 \zeta^{2}\right)}{25+64 \alpha^{2}}\right. \\
& \left.-16 \alpha^{2} \frac{1665+2432 \alpha^{2}}{25+64 \alpha^{2}} \cdot \frac{r}{h} \frac{\mathrm{d} h}{\mathrm{~d} r}+192 \alpha^{2}\left(\frac{r}{h} \frac{\mathrm{d} h}{\mathrm{~d} r}\right)^{2}\right]
\end{aligned}
$$

\section{Appendix B: Detailed calculations for $O\left(\epsilon^{2}\right)$ dynamics}

\section{B.1. Vertical acoustics}

We begin this analysis with an exploration of the dynamics arising from the solution to the homogeneous part of Eq. (39),

$\mathcal{L} v_{\mathrm{h}}^{\prime}=0$. 
Recalling the arguments pointed out in the text, in this circumstance the vertical velocity and density fluctuations are completely decoupled from the horizontal (radial and azimuthal) motions. It is in this context that we consider the simplest vertical motions possible, Given that there are no horizontal velocities induced by these motions, the boundary condition (29) reduces to

$$
\left.P_{0} \frac{\partial v_{\mathrm{h}}^{\prime}}{\partial z}\right|_{z=h}=0 \text {. }
$$

Assuming the solution Ansatz (48) we find that Eq. (B.1) reduces to

$$
\begin{aligned}
& -\frac{1}{n}\left(1+\frac{8}{9} \frac{\tilde{\sigma} \alpha n}{n+1}\right)\left[\left(1-\zeta^{2}\right) \frac{\partial^{2}}{\partial \zeta^{2}}\right. \\
& \left.-2(n+1) \zeta \frac{\partial}{\partial \zeta}-2 n \frac{\tilde{\sigma}^{2}+1}{1+\frac{8}{9} \frac{\tilde{\sigma} \alpha n}{n+1}}\right] \hat{v}_{\mathrm{h}}^{\prime}=0,
\end{aligned}
$$

where for convenience we have introduced the height scaled variable $\zeta \equiv z / h$.

It is worth reviewing the behavior of possible solutions to the operator (B.3) near the singular points $\zeta= \pm 1$. In particular, if we assume that the solution functionally behaves according to the form

$\hat{v}_{\mathrm{h}}^{\prime} \sim\left(1-\zeta^{2}\right)^{\lambda}$

then an indicial equation analysis (Morse \& Feshbach 1953) shows that to leading order there are two values of $\lambda$ (corresponding to two linearly independent solutions) which are 0 and $-n$. The former choice tells us that there is, in fact, a solution described by a regular series expansion near \pm 1 . As for the latter solution, since it predicts that $\hat{v}_{\mathrm{h}}^{\prime}$ diverges like $\zeta^{-n}$ the product $P_{0} \partial \hat{v}_{\mathrm{h}}^{\prime} / \partial z$ none the less remains constant. However this solution must still be rejected because it would cause the zero Lagrangian pressure condition on the surface to be violated, i.e. Eq. (44) would not be satisfied.

As it turns out, the solution to Eq. (B.3) is composed of the associated Legendre polynomials $P$ and $Q$ (Abramowitz \& Stegun 1972) as follows,

$\hat{v}_{\mathrm{h}}^{\prime}=\frac{\tilde{A}(r)}{(1-\zeta)^{n / 2}} \cdot\left[P_{\Delta}^{n}(\zeta) Q_{\Delta}^{n}(0)-Q_{\Delta}^{n}(\zeta) P_{\Delta}^{n}(0)\right]$,

with

$$
\begin{aligned}
\Delta(\tilde{\sigma}, n, \alpha) \equiv & \frac{1}{2}\left\{-1+\left[1+\left((2 n+1)^{2}\right.\right.\right. \\
& \left.\left.\left.-\frac{8 n(n+1)\left(1+\tilde{\sigma}^{2}\right)}{\left.n+1+\frac{8}{9} n \alpha \tilde{\sigma}\right)}\right)\right]^{1 / 2}\right\},
\end{aligned}
$$

and this solution satisfies the condition (44). The $z$ independent constant $\tilde{A}(r)$ is entirely arbitrary and is determined by the initial conditions. In order to satisfy the boundary condition at $z=h$ a somewhat complicated expression emerges for the eigenvalue condition. However, it turns out that for special cases of the polytropic index the quantization condition for these modes may be easily written down, namely, for $n$ a positive integer or half odd integer it is required that,

$\Delta=2 k+n+1$,

where $k$ is any integer $k \geq 0$. In this sense $k$ labels the acoustic overtone of the mode with $k=0$ being the fundamental. Given the definition of $\Delta$ above, for every $k$ there are two values of $\tilde{\sigma}$ which are always decaying. In considering all fundamental modes, which we label with $\tilde{\sigma}_{\mathrm{F}}$, we find that,

$$
\begin{aligned}
\tilde{\sigma}_{\mathrm{F}} & =-\frac{4}{9} \alpha \pm\left(\frac{16}{81} \alpha^{2}-\frac{2 n+1}{n}\right)^{1 / 2} \\
& \longrightarrow-\frac{4}{9} \alpha \pm \mathrm{i}\left|\left(\frac{16}{81} \alpha^{2}-\frac{2 n+1}{n}\right)\right|^{1 / 2}, \text { for } \alpha<\alpha_{\mathrm{osc}}
\end{aligned}
$$

It is interesting to note that for $\alpha<\alpha_{\mathrm{osc}}$, given by,

$\alpha_{\mathrm{osc}}=\frac{9}{4} \sqrt{\frac{2 n+1}{n}}$,

the modes are complex conjugate pairs of decaying oscillations. For $\alpha>\alpha_{\text {osc }}$ the modes are strictly exponentially decaying. For example, for $n=3 / 2, \alpha_{\text {osc }}=(9 / 4) \cdot \sqrt{8 / 3} \approx 4$. Furthermore, in the zero viscosity limit, the oscillation frequency is given by

$\tilde{\sigma}_{\mathrm{F}}(\alpha \rightarrow 0)= \pm \mathrm{i} \sqrt{\frac{2 n+1}{n}}$.

\section{B.2. General driven acoustics}

We now turn to solving the coupled Eqs. (38), (39). Given the structure of these equations we must begin with developing a solution to Eq. (38) and then using this in Eq. (39). We suppose, as we did in the previous section, that the solution to Eq. (38), which is a homogeneous equation, has the following form,

$u_{1}^{\prime}=\hat{u}^{\prime}(z, r) \mathrm{e}^{\sigma T}+$ c.c.,

where the scaled time is defined as $T \equiv t / r^{3 / 2}$ (as in the text). We write the eigenvalue as $\sigma$ in order to distinguish it from the "vertical acoustics" eigenvalue, $\tilde{\sigma}$, discussed in the previous section. After a little manipulation we find that Eq. (38) turns into the far simpler ODE

$\Omega_{0}^{2}\left\{\left[\sigma-\frac{\alpha}{2(n+1)}\left(1-\zeta^{2}\right) \frac{\partial^{2}}{\partial \zeta^{2}}+\alpha \zeta \frac{\partial}{\partial \zeta}\right]^{2}+1\right\} \hat{u}^{\prime}=0$.

Before presenting the Ansatz for the solution it is worthwhile to consider the array of solution behavior of Eq. (B.8) near the singular points $\zeta= \pm 1$. Assuming that near $\zeta=1$ the behavior is $\hat{u}^{\prime} \sim\left(1-\zeta^{2}\right)^{\beta}$, an indicial analysis (Morse \& Feshbach 1953) reveals that there are four choices for $\beta$, namely, $0,1,-n, 1-n$. The solutions with $\beta=-n, 1-n$ are rejected on grounds that they violate the boundary condition at $\zeta= \pm 1$ (they essentially diverge). In other words, since $\eta$ is proportional to $P_{0}$, then as one approaches the boundary, $\eta\left(\partial u^{\prime} / \partial z\right), \eta r\left(\partial \Omega^{\prime} / \partial r\right) \neq 0$ there for these two values of $\beta$. It would then mean that the stress conditions (45) would be violated there and so these solutions are ignored. The remaining two possibilities suggest that a regular 
truncated series expansion is a satisfactory means of expressing the solution to $u^{\prime}$.

Thus, for varicose modes we assume the Ansatz

$\hat{u}^{\prime}=\hat{u}_{(k)}=\sum_{m=0}^{k+1} A_{m}(r) \zeta^{2(k+1)-2 m}$,

where $k$ labels the eigenmode and is an integer greater than or equal to zero. Correspondingly, the eigenvalue, $\sigma$, would be labelled by $k$ too. Aside from a generalized characterization of the eigenvalues for arbitrary $k$ (see below), from here on we restrict ourselves to considering only the fundamental mode, i.e. $k=0$ and thus,

$\hat{u}^{\prime}=\hat{u}_{(0)}=A(r) \zeta^{2}+B(r)$,

where the constants have been redefined for simplicity. Generally speaking, insertion of the solution form (B.9) into Eq. (B.8) requires one to recursively solve for the values of the coefficients $A_{k}$ in the usual procedure called for when developing series solutions to ODE's. The first of these equations is always a relationship for the eigenvalue $\sigma$. For example inserting Eqs. (B.10) into (B.8) gives

$A\left[\left(\sigma+\alpha \frac{2}{3} \frac{2 n+3}{n+1}\right)^{2}+1\right] \zeta^{2}+\sigma\left(\sigma B-A \frac{2}{3} \frac{\alpha}{n+1}\right)$
$-A \frac{2}{3} \frac{\alpha}{n+1}\left(\sigma+\frac{2}{3} \alpha \frac{2 n+3}{n+1}\right)+B=0$,

and by setting to zero the coefficients of each power of $\zeta$ one gets

$\sigma=\sigma_{ \pm}= \pm \mathrm{i}-\alpha \frac{2 n+3}{n+1}, \quad \frac{B(r)}{A(r)}=-\frac{1}{2 n+3}$.

In other words, there are the two possible temporal responses (denoted by $\sigma_{ \pm}$), to this type of disturbance, however, the coefficients are the same for both. We note that the eigenvalue form indicates that these modes exhibit decaying oscillations. For general values of $k$, the frequency response obtained by inserting Eqs. (B.9) into (B.8), is given by

$\sigma_{k}=\sigma(k)= \pm \mathrm{i}-\frac{(k+1)(2 n+3+2 k)}{(n+1)} \frac{2}{3} \alpha$,

showing that these modes become increasingly damped in proportion to $k^{2}$ in the large $k$ limit. The general solution for an arbitrary disturbance can thus be written as

$u_{1}^{\prime}=\sum_{k=0}^{\infty} \hat{u}_{(k)}(\zeta, r) \mathrm{e}^{\sigma_{k} T}+$ c.c.

We note also that the integrated radial flux of mass for the perturbation vanishes, namely that,

$\int_{-1}^{1} \rho_{0} r \hat{u}^{\prime} \mathrm{d} \zeta=0$

and this is generally true for any integer mode $k$. The consequence being, as will be seen below, that the radial structure of the perturbation amplitude, namely the quantity $A(r)$, remains a freely chosen function, usually guided by some initial condition.
Now we turn to expanding the particular solution to Eq. (39) armed with the solutions (B.7), (B.10) and (B.11). Inspection of the operator structures of $\mathcal{L}, \mathcal{F}$ and $\mathcal{G}$ suggests that the solution to the particular vertical velocity, $v_{\mathrm{p}}^{\prime}$, has the form

$v_{\mathrm{p}}^{\prime}=\sum_{k=0}^{\infty}\left(\hat{v}_{(k)}(\zeta, r) \mathrm{e}^{\sigma T}+\hat{V}_{(k)}(\zeta, r) T \mathrm{e}^{\sigma T}\right)+$ c.c.,

in which

$\hat{v}_{(k)}=\sum_{m=0}^{k+1} \Xi_{k, 2 m}(r) \zeta^{2(k+1)-2 m+1}$,

$\hat{V}_{(k)}=\sum_{m=0}^{k+1} \Theta_{k, 2 m}(r) \zeta^{2(k+1)-2 m+1}$.

We note that these solutions have terms which manifest TG accompanied by oscillations due to the form $t \mathrm{e}^{-\alpha t} \mathrm{e}^{\mathrm{i} \omega t}$ type of behavior. Recalling that we are here only considering the fundamental mode, the above solution form is written as

$\hat{v}_{(0)}=\Xi_{00}(r) \zeta^{3}+\Xi_{02}(r) \zeta, \quad \hat{V}_{(0)}=\Theta_{00}(r) \zeta^{3}+\Theta_{02}(r) \zeta$.

Using Eq. (B.16) together with the solutions (B.7), (B.10) in Eq. (39) and after some lengthy algebra (which was verified using Mathematica 5.0), the coefficients straightforwardly follow. Unfortunately, their expressions are very long and we have omitted their presentation here. This solution for $v_{\mathrm{p}}^{\prime}$ along with the solution for $u_{1}^{\prime}$, when used in Eq. (36) immediately yields the solution to $\rho_{2}^{\prime}$. Its expansion is similar to that of $v_{\mathrm{p}}^{\prime}$, namely, we say that $\rho_{2}^{\prime} \sim \rho_{\mathrm{p}}^{\prime}+\rho_{\mathrm{h}}^{\prime}$, representing its particular and homogeneous contributions. Then it follows that

$\rho_{\mathrm{p}}^{\prime}=\hat{\rho}^{\prime}(\zeta, r) \mathrm{e}^{\sigma T}+\hat{R}^{\prime}(\zeta, r) T \mathrm{e}^{\sigma T}+$ c.c.

in which

$\hat{\rho}^{\prime}=\hat{\rho}_{(k)}=\sum_{m=0}^{k+2} \Xi_{k, 2 m}^{(\rho)}(r) \zeta^{2(k+1)-2 m}$,

$\hat{R}^{\prime}=\hat{R}_{(k)}=\sum_{m=0}^{k+2} \Theta_{k, 2 m}^{(\rho)}(r) \zeta^{2(k+1)-2 m}$

The superscript $(\rho)$ appearing in the above expressions is meant as a label to distinguish these functions from the corresponding ones for the vertical velocity mode, $v_{\mathrm{p}}^{\prime}$, cf. Eq. (B.15). The lowest density fluctuation eigenmode is

$\hat{\rho}_{(0)}=\Xi_{00}^{(\rho)}(r) \zeta^{4}+\Xi_{02}^{(\rho)}(r) \zeta^{2}+\Xi_{04}^{(\rho)}(r)$,

$\hat{R}_{(0)}=\Theta_{00}^{(\rho)}(r) \zeta^{4}+\Theta_{02}^{(\rho)}(r) \zeta^{2}+\Theta_{04}^{(\rho)}(r)$.

The solutions quoted in the text have this form. We note also that the boundary conditions on the vertical velocity components, namely,

$\left(1-\zeta^{2}\right)^{n+1}\left[\frac{1}{h(r)} \frac{\partial \hat{v}_{\mathrm{p}}^{\prime}}{\partial \zeta}+\frac{1}{r} \frac{\partial r \hat{u}_{1}^{\prime}}{\partial r}\right]=0$,

as $\zeta \rightarrow 1$, is satisfied by these solutions. 


\section{References}

Abramowitz, M., \& Stegun, I. 1972, Handbook of Mathematical Functions (New York: Dover)

Afshordi, N., Mukhopadhyay, B., \& Narayan, R. 2005, ApJ, 629, 373

Balbus, S. A., \& Hawley, J. F. 1991, ApJ, 376, 214

Balbus, S. A., Hawley, J. F., \& Stone, J. M. 1996, ApJ, 467, 76

Balbus, S. A. 2003, ARA\&A, 41, 555

Balmforth, N. J., \& Spiegel, E. A. 1996, Physica D, 97, 1

Barranco, J., \& Marcus, P. S. 2005, ApJ, 623, 1157

Chagelishvili, G. D., Zahn, J.-P., Tevzadze, A. G., \& Lominadze, J. G. 2003, A\&A, 402, 401

Dubrulle, B., Marie, L., Normand, Ch., Richard, D., Hersant, F., \& Zahn, J.-P. 2004, A\&A, accepted [arXiv: astro-ph/0410224]

Criminale, W. O., Jackson, T. L., \& Joslin, R. D. 2003, Theory and Computation in Hydrodynamics Stability (Cambridge: Cambridge Univ. Press)

Frank, J., King, A. R., \& Raine, D. J. 2002, Accretion Power in Astrophysics (Cambridge: Cambridge Univ. Press)

Fromang, S., Terquem, C., \& Balbus, S. A. 2002, MNRAS, 329, 18

Grossman, S. 2000, Rev. Mod. Phys., 72, 603

Hanifi, A., Schmid, P. J., \& Henningson, D. S. 1996, Phys. Fluids, 8, 826

Hoshi, R. 1977, Progr. Theor. Phys., 58, 1191

Ioannou, P. J., \& Kakouris, A. 2001, ApJ, 550, 931

Klahr, H. H., \& Bodenheimer, P. 2003, ApJ, 582, 869

Kluźniak, W., \& Kita, D. 2000, Three-dimensional structure of an alpha accretion disk [arXiv: astro-ph/0006266]

Landau, L. D., \& Lifshitz, E. M. 1989, Fluid Mechanics, 2nd Ed. (Burlington MA: Elsevier B-H)

Lesur, G., \& Longaretti, P.-Y. 2005, preprint

Lin, D. N. C., \& Papaloizou, J. C. B. 1996, ARA\&A, 34, 703

Longaretti, P.-Y. 2002, ApJ, 587

Lynden-Bell, D., \& Pringle, J. E. 1974, MNRAS, 168, 603

Menou, K. 2000, Science, 288(5473), 2022
Molemaker, J. J., McWilliams, J. C., \& Yavnew, I. 2001, Phys. Rev. Lett., 86, 5273

Monin, A. S., \& Yaglom, A. M. 1971, Statistical Fluid Mechanics (Cambridge MA: MIT Press)

Morse, P. M., \& Feschbach, H. 1953, Methods of Theoretical Physics (New York: McGraw-Hill)

Mukhopadhyay, B., Afshordi, N., \& Narayan, R. 2005, ApJ, 629, 383

Papaloizou, J. C. B., \& Lin, D. N. C. 1995, ARA\&A, 33, 505

Pringle, J. E. 1981, ARA\&A, 19, 137

Rayleigh, J. W. S. 1877, The Theory of Sound (NY: Dover Publications), reissued 1976

Regev, O. 1983, A\&A, 126, 146

Regev, O., \& Bertout, C. 1995, MNRAS, 272, 71

Regev, O., \& Gitelman, L. 2002, A\&A, 396, 623

Rudiger, G., Arlt, R., \& Shalybkov, D. 2002, A\&A, 391, 781

Sano, T., Miyama, S. M., Umebayashi, T., \& Nakano, T. 2000, ApJ, 543,486

Schmid, P. J., \& Henningson, D. S. 2001, Stability and transition in shear flows (New York: Springer)

Shakura, N. I., \& Sunyaev, R. A. 1973, A\&A, 24, 337

Shalybkov, D., \& Rudiger, G. 2005, A\&A, 438, 411

Tassoul, J. 2000, Stellar rotation (Cambridge: Cambridge University Press)

Tevzadze, A. G., Chagelishvili, G. D., Zahn, J.-P., Chanishvili, R. G., \& Lominadze, J. G. 2003, A\&A, 407, 779

Umurhan, O. M. 2005, On the stratorotational instability in the semi-hydrostatic quasi-geostrophic limit, MNRAS, accepted

Umurhan, O. M., \& Regev, O. 2004, A\&A, 427, 855

Umurhan, O. M., \& Shaviv, G. 2005, A\&A, 432, L31

Vishniac, E. T., \& Park, S. J. 2005, Astrophysical Accretion Disks, Frontiers in Physics Series - Basic Books, New York

Yavneh, I., McWilliams, J. C., \& Molemaker, M. J. 2001, J. Fluid Mech., 448, 1

Yecko, P. A. 2004, A\&A, 425, 385 